\title{
On Universal Modeling of the Bulk Acoustic Wave Devices
}

\author{
Boris Sveshnikov, Sergey Nikitov and Sergey Suchkov
}

Additional information is available at the end of the chapter

http://dx.doi.org/10.5772/53191

\section{Introduction}

Delay lines, as well as thin film acoustic resonators (FBAR) and solidly mounted resonators (SMR) of bulk acoustic waves (BAW), are widely used in the modern wireless communication networks due to their compactness and ability to operate in the ultra-high and super-high frequencies.

The most rigorous description of the characteristics of arbitrary BAW devices may be achieved by using the known three dimensional finite element method (e.g., Giraud et al, 2007). However, this approach demands enormous computational resources and a long computation time during each design of BAW resonators based on multilayer topologies. So, it is difficult to optimize the system construction promptly, with respect to the peculiarities of each particular specification.

In addition, the results obtained by means of FEM analysis do not have a clear physical sense. Consequently, every time it is not evident which factors of either material or constructive nature influence dominantly on the degradation of the real system performances, and one has to make rather expensive experiments to answer the necessary questions in the course of optimization of a concrete design. Thus, an evident interest appears to develop approximate but flexible analytical approach, allowing fast preliminary investigation and synthesis of any BAW system ${ }^{1}$.

We would like to restrict our theoretical investigation here to the one-dimensional (1D) analysis: the model described below admits changes of the system properties along the longitudinal coordinate only. This approach doesn't merely simplify our analysis: it has also some objective advantages in the sense of its practical use due to the following.

1 3D-FEM approach may be aplyied at the final stage of needed design, if necessary. 
In general, BAW devices represent a set of layers made of various materials. Where parallel facets that are perpendicular not only to the "working" X-direction, but also to $\mathrm{Y} \& \mathrm{Z}$ axes exist (Fig.1a), the synchronous resonant excitations of spurious lateral modes associated with the simultaneous excitations of transverse bending wave motion in plates with side edges parallel to one another become inevitable. The main shortcoming of one-dimensional models is their inability to take into account only those spurious lateral modes.

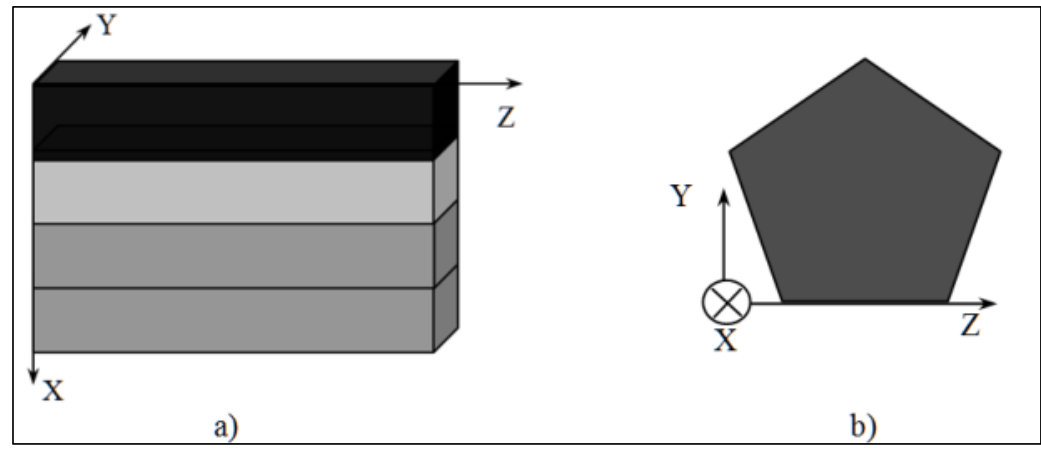

Figure 1. Schematic of layered BAW system with parallel planes being perpendicular to $X, Y, \& Z$ axes, where the desired bulk acoustic waves propagate along X-direction (a), and the preferred configuration of the "top-side" electrode (Bradley et al., 2002) in an actual BAW device (b).

However, a usage of irregular polygon electrode, in which no two sides are parallel to one in the directions being perpendicular to Y- \& Z- axes (Fig.1b), allows considerable suppression of parasitic movements in similar systems (Bradley et al., 2002). Thus, we must only introduce - on the phenomenological level - the proper imaginary addends to the wavenumbers in every layer, taking into account the losses caused by non-synchronous excitation and transformation to heat of parasitic modes (in addition to propagation losses of acoustic waves because of the material viscosity).

Therefore, a one-dimensional model, which allows involving additional losses into analysis, is even more suitable for studying real BAW devices, than, say, two-dimensional models, which demand much more complex routine but cannot be used in design of actual devices with polygonal electrodes.

It is noteworthy that one-dimensional simulation of BAW device is a preferable and correct approach if the direction of the wave propagation in a crystal coincides with the axis of its symmetry. This is so at least in the case of the widely used orientations of $\mathrm{ZnO}, \mathrm{AlN}, \mathrm{W}, \mathrm{Mo}$, $\mathrm{SiO}_{2}, \mathrm{Ti}, \mathrm{Al}, \mathrm{Al}_{2} \mathrm{O}_{3}$, $\mathrm{Si}$, etc.

Despite numerous publications on research of BAW devices even in a simplified onedimensional case (e.g., Hashimoto, K. (Ed.), 2009), to date no-one has provided reliable fast calculations of many real systems with complex structures. For example, an equivalent circuit network analysis (Ballato et al., 1974) becomes progressively difficult with increased number of electrodes. On the other hand, the most general modelling approach, based on 
the direct solution of the relevant equations for electro-acoustic fields (Novotny, H., et al., 1991), cannot describe adequately many different configurations of practical importance (e.g., a real SMR). Moreover, Novotny's model is based on a cumbersome cascading routine, where every layer is being represented by a $(8 \times 8)$ transfer matrix. Accordingly, it is difficult to arrange enough complex electrical circuitry in that manner while minimizing at the same time inevitable mistakes during preparation of corresponding software tools.

The novel one-dimensional theory is a more universally suitable designing tool, since it is both much clearer and simpler in use than predecessors. At the same time an effective selfchecking algorithm based on satisfaction of three fundamental conditions (energy balance, the second law of thermodynamics and reciprocity) is proposed and utilized in the application software.

Applying the newly developed approach, one would be capable of analyzing - while remaining in the frame of the same modelling principles - any system with an arbitrary number and sequence of dielectric and metal layers under arbitrary inter-electrode connections ${ }^{2}$. Multiple electrodes may compose the multilayer transducers, forming either those based one-port and two-port networks, or tunable re-radiators, loaded by variable admittance. The last variant may be used in order to control electrically the frequency responses of various modern devices, based on usage of bulk acoustic waves.

\section{Viscous losses in BAW devices}

One of the most important aspects affecting the quality of real BAW devices, is the energy losses which emerge during propagation of acoustic waves in crystals. The main cause of the wave attenuation in this case is a viscosity of elastic medium, i.e., the friction arising due to the mechanical movements of the material particles with respect to the neighboring environment. A reliable quantitative estimation of propagation losses is possible only on the basis of experiments.

Many similar experiments have been carried out earlier by different groups of researchers (e.g., Gulyaev, Yu., \& Mansfeld, G., 2004). Their results show that the logarithmic decrement characterizing propagation loss per BAW's wavelength depends on frequency almost linearly and can be expressed as $\zeta(f)=\zeta_{1} \cdot f$. Table 1 shows, for example, a set of $\zeta_{1}$ - values, related to some materials, commonly used in modern BAW resonators.

\begin{tabular}{|l|l|l|l|l|l|l|l|l|l|l|l|l|}
\hline Mat. & $\mathrm{Al}$ & $\mathrm{AlN}$ & $\mathrm{ZnO}$ & $\mathrm{SiO}_{2}$ & $\mathrm{~W}$ & $\mathrm{Ti}$ & $\mathrm{Mo}$ & $\mathrm{Si}$ & $\mathrm{Al}_{2} \mathrm{O}_{3}$ & Diam & LTO & LNO \\
\hline $\begin{array}{l}\zeta_{1} \cdot 10^{3} \\
(1 / \mathrm{GHz})\end{array}$ & 4.13 & 0.58 & 1.04 & 0.15 & 1.74 & 4.9 & 1.16 & 0.86 & 0.025 & 0.45 & 0.0058 & 0.014 \\
\hline
\end{tabular}

Table 1. Values of logarithmic decrement $(1 / \mathrm{GHz})$, evaluating attenuation per wavelength of BAW in some popular materials.

\footnotetext{
${ }^{2}$ The infinite conductivity of electrodes is assumed below, when free charges may concentrate only in the infinitely thin skins at metal's borders.
} 


\section{Simulation principles}

In order to calculate the characteristics of multilayer system, we should consider within each layer the motion equation and constitutive relations (Auld, 1973), reducing them to the onedimensional case in application to the selected bulk wave mode - either longitudinal or the shear one (Kino, 1987).

Let us denote the normal component of the elastic stress tensor as $T(\mathrm{~Pa})$, while $u=$ elastic displacement $(m), \rho=$ mass density $\left(\mathrm{kg} / \mathrm{m}^{3}\right), D=$ electric displacement $\left(\mathrm{C} / \mathrm{m}^{2}\right)$ in the presence of electric field with intensity $E(V / m)$. Besides, $c, \beta, \varepsilon$, and $\eta$ mean, respectively, the corresponding components of tensors, characterizing elastic stiffness $(\mathrm{Pa})$, piezoelectric stress $\left(\mathrm{C} / \mathrm{m}^{2}\right)$, relative permittivity $(\mathrm{F} / \mathrm{m})$, and viscosity $(\mathrm{s} \cdot \mathrm{Pa})$.

Then, a well known system of the motion equation (1) and constitutive relations (2-3) should be written for each layer (Auld, 1973):

$$
\begin{gathered}
\frac{\partial \mathrm{T}}{\partial \mathrm{x}}=\mathrm{Q} \cdot \frac{\partial^{2} \mathrm{u}}{\partial \mathrm{t}^{2}} \\
T=c \cdot \frac{\partial u}{\partial x}+\eta \cdot \frac{\partial^{2} u}{\partial x \partial t}-\beta \cdot E \\
D=\varepsilon \cdot E+\beta \cdot \frac{\partial u}{\partial x}
\end{gathered}
$$

At this point a quasi-static approximation of Maxwell's equations holds everywhere, except neighbor edges of the electrodes with different polarity:

$$
\frac{\partial D_{j}}{\partial x}=0
$$

It is convenient to convert (2-3) to the following relations for the electric intensity and elastic stress:

$$
\begin{gathered}
E=\frac{1}{\varepsilon} \cdot\left(D-\beta \cdot \frac{\partial u}{\partial x}\right) \\
T=\tilde{c} \cdot \frac{d u}{d x}-\frac{\beta}{\varepsilon} \cdot D
\end{gathered}
$$

The following denotations are used here:

$q=\omega \cdot \sqrt{\frac{\rho}{\tilde{c}}}, \quad \tilde{c}=c\left(1+k^{2}\right)+i \omega \eta=c \cdot\left(1+k^{2}\right) \cdot\left(1+i \cdot \frac{\zeta}{\pi}\right), \quad k^{2}=\frac{\beta^{2}}{\varepsilon \cdot c}, \quad$ while the coefficient $\zeta=\pi \frac{\omega \eta}{c \cdot\left(1+k^{2}\right)}$ is a logarithmic decrement, characterizing total distributed dissipation per wavelength of bulk acoustic waves in the chosen medium. 
NB: $v=\sqrt{\frac{c \cdot\left(1+k^{2}\right)}{\rho}}$ is a BAW velocity in the lossless case.

Assuming, as usual, a harmonic solution and omitting the time oscillating factor $e^{i \omega t}$, one can simply obtain from Eqs. $(1,4,6)$ the wave equation for dissipative medium:

$$
\frac{\partial^{2} u}{\partial x^{2}}+q^{2} \cdot u=0
$$

Therefore, it is possible to find a solution in every $j$-th layer, i.e. within a spatial interval $x_{j-1} \leq$ $x \leq x_{j}(j=1,2 \ldots N)$, as a superposition of two counterpropagating waves:

$$
u_{j}(x)=U_{j} \cdot e^{-i q_{j} \cdot\left(x-x_{j-1}\right)}+U_{j+N} \cdot e^{i q_{j} \cdot\left(x-x_{j-1}\right)}
$$

In analogy with the previously developed approach to study arbitrary BAW devices, we are using below the sequential "end-to-end" subscripting of wave amplitudes: index of the backward wave comes to hand from an index of the forward wave within the same layer simply by adding $N$-figure, where $N$ is a number of the system layers, including electrodes and substrate (Sveshnikov, 2009). Amplitudes $U_{m}(m=1,2 \ldots 2 N)$ have to be found when satisfying all the boundary conditions at the layer interfaces $x_{j}$ :

$$
\begin{gathered}
u\left(x_{j}-0\right)=u\left(x_{j}+0\right) \\
T\left(x_{j}-0\right)=T\left(x_{j}+0\right) \\
D\left(x_{j}+0\right)-D\left(x_{j}-0\right)=\sigma\left(x_{j}\right)
\end{gathered}
$$

where $\sigma\left(x_{j}\right)$ means the surface charge density on $j$-th interface ${ }^{3}$.

Before giving the general solution of the stated problem, we'd like to describe, first of all, solutions of a few typical tasks to facilitate understanding of the present modelling logics.

\subsection{P-matrix of a circuitry containing a single piezoelectric layer}

Let us describe a simple bulk acoustic wave transducer (BT), formed by a piezoelectric layer with thickness ' $d$ ', placed between perfectly conductive metal electrodes, infinitely extended along the acoustic channel (Fig.2). This structure may be used either as a transducer directly ( $\mathrm{S}$ is in position "1"), or as a tunable reflector loaded by variable admittance Yo (S $\Leftrightarrow$ "2").

As a three-port network containing one electrical and two acoustic ports it may be characterized by means of the usual $P$-matrix. Its sense is explained by the following relations, appropriate when switch $S$ on Fig. 2 is in the $1^{\text {st }}$ position:

${ }^{3} \sigma_{j}$ - values differ from zero only at the edges of neighbor electrodes with different polarity. 


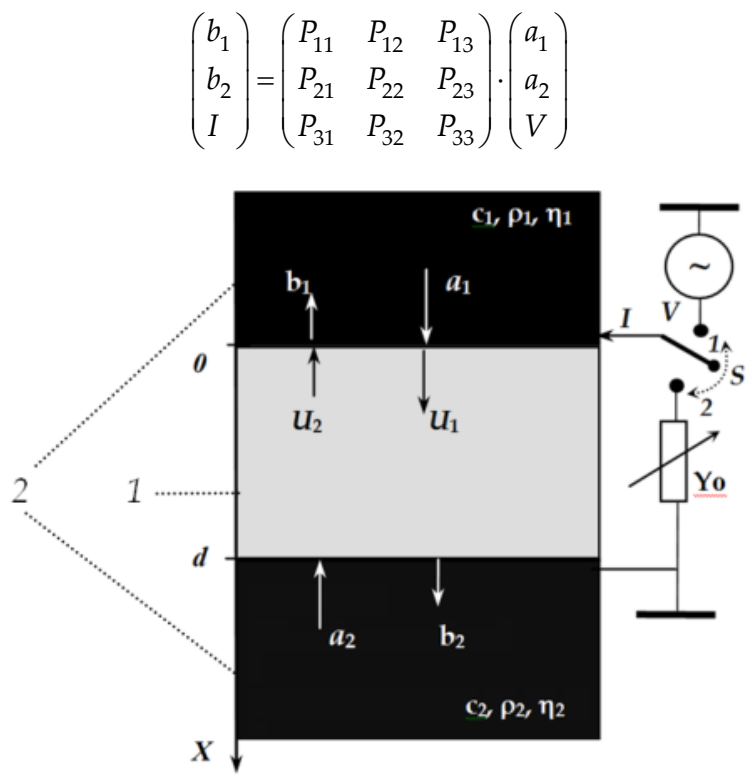

Figure 2. A piezoelectric layer (1), placed between two semi-infinite electrodes (2), made, in general, from different materials (we assume below that they are perfectly conductive).

One can find all needed terms of this $P$-matrix in two steps.

\subsection{1. "One-layer" transducer of bulk acoustic waves}

Assuming that there no beams launching upon a BT from outside $\left(a_{1}=a_{2}=0\right.$, satisfaction of the boundary conditions (9-11) at the cross-sections $x=0 \& x=d$ gives us a set of linear equations for five unknowns:

$$
\begin{gathered}
U_{1}+U_{2}=b_{1} \\
-i \cdot \tilde{c} q\left(U_{1}-U_{2}\right)-\frac{\beta}{\varepsilon} D=i \tilde{c}_{1} q_{1} \cdot b_{1} \\
U_{1} \cdot e^{-2 i \psi}+U_{2} \cdot e^{2 i \psi}=b_{2} \\
-i \cdot \tilde{c} q\left(U_{1} \cdot e^{-2 i \cdot \psi}-U_{2} \cdot e^{2 i \cdot \psi}\right)-\frac{\beta}{\varepsilon} D=-i \tilde{c}_{2} q_{2} \cdot b_{2} \\
D=\frac{\varepsilon}{d} V-i \beta \cdot q \cdot \frac{\sin (\psi)}{\psi} \cdot\left(U_{1} \cdot e^{-i \cdot \psi}-U_{2} \cdot e^{i \cdot \psi}\right)
\end{gathered}
$$$$
\text { Here } D=D(0+)=D(d-0) \text { and } \psi=q \cdot d / 2 \text {. }
$$ 
Note that equality (17) is obtained by integrating (5) over a piezoelectric layer:

$$
\int_{0}^{d} E(x) \cdot d x=V
$$

When substituting (13) \& (15) into (14) \& (16), one can get a couple of equations allowing us to express the amplitudes $U_{1,2}$ through applied voltage $V$ :

$$
\left\{\begin{array}{c}
\left(1+\frac{Z_{1}}{Z}-K_{e}^{2} \cdot \frac{\sin (\psi)}{\psi} \cdot e^{-i \cdot \psi}\right) \cdot U_{1}-\left(1-\frac{Z_{1}}{Z}-K_{e}^{2} \cdot \frac{\sin (\psi)}{\psi} \cdot e^{i \cdot \psi}\right) \cdot U_{2}=\frac{i \beta \cdot V}{\tilde{c} \cdot q \cdot d} \\
\left(1-\frac{Z_{2}}{Z}-K_{e}^{2} \cdot \frac{\sin (\psi)}{\psi} \cdot e^{i \cdot \psi}\right) \cdot e^{-2 i \cdot \psi} \cdot U_{1}-\left(1+\frac{Z_{2}}{Z}-K_{e}^{2} \cdot \frac{\sin (\psi)}{\psi} \cdot e^{-i \cdot \psi}\right) \cdot e^{2 i \cdot \psi} \cdot U_{2}=\frac{i \beta \cdot V}{\tilde{c} \cdot q \cdot d} \\
Z_{j}=\sqrt{\rho_{j} \cdot \tilde{c}_{j}}=\frac{\tilde{c}_{j} q_{j}}{\omega}
\end{array}\right.
$$

Parameter $Z_{j}$ above has a sense of acoustic impedance (Pa.s $/ \mathrm{m}$ ) of $j$-th layer (subscript is omitted for a middle film), being complex valued quantity in the presence of dissipation, and $K_{e}^{2}=\frac{k^{2}}{1+k^{2}}$ is an effective piezoelectric coupling constant (Kino, 1987).

By substituting solutions $U_{1,2}$ of (19) to (13) \& (15), we find the terms $P_{23,13}$ of the considered $P$-matrix, characterizing amplitudes of the "forward" and "backward" acoustic beams, radiated into the neighboring semi-infinite acoustic media.

$$
P_{23,13}=\frac{ \pm \frac{\beta}{\tilde{c} \cdot q \cdot d} \cdot \tan (2 \psi)\left(\frac{Z_{1,2}}{Z}+i \cdot \tan (\psi)\right)}{\frac{Z_{1}+Z_{2}}{Z}\left(1-K_{e}^{2} \cdot \frac{\tan (2 \psi)}{2 \psi}\right)+i \cdot \tan (2 \psi)\left(1+\frac{Z_{1} Z_{2}}{Z^{2}}-K_{e}^{2} \cdot \frac{\tan (\psi)}{\psi}\right)}
$$

On the other hand, with the help of (17) one can calculate an admittance of considered "onelayer" transducer as $P_{33}=i \cdot \omega \cdot D(0<x<d) \cdot S / V$ :

$$
P_{33}=\frac{i \cdot \omega \cdot C_{0} \cdot\left\{\frac{Z_{1}+Z_{2}}{Z}+i \cdot \tan (2 \psi) \cdot\left(1+\frac{Z_{1} Z_{2}}{Z^{2}}\right)\right\}}{\frac{Z_{1}+Z_{2}}{Z}\left(1-K_{e}^{2} \cdot \frac{\tan (2 \psi)}{2 \psi}\right)+i \cdot \tan (2 \psi)\left(1+\frac{Z_{1} Z_{2}}{Z^{2}}-K_{e}^{2} \cdot \frac{\tan (\psi)}{\psi}\right)^{\prime}},
$$

where $C_{0}=\frac{\varepsilon \cdot S}{d}$ is the static capacitance of a BT, if an area of its electrodes equals $S$. In the lossless case $(\zeta=0)$ conductance of similar transducer $G a=\operatorname{Re}\left(\mathrm{P}_{33}\right)$ is expressed as follows: 


$$
G a=\frac{\omega \cdot C_{0} \cdot K_{e}^{2} \cdot \frac{Z_{1}+Z_{2}}{Z} \cdot \frac{(\tan (2 \psi))^{2}}{2 \psi} \cdot\left\{\frac{Z_{1} Z_{2}}{Z^{2}}+(\tan (\psi))^{2}\right\}}{\left\{\frac{Z_{1}+Z_{2}}{Z}\left(1-K_{e}^{2} \cdot \frac{\tan (2 \psi)}{2 \psi}\right)\right\}^{2}+\left\{\tan (2 \psi)\left(1+\frac{Z_{1} Z_{2}}{Z^{2}}-K_{e}^{2} \cdot \frac{\tan (\psi)}{\psi}\right)\right\}^{2}}
$$

In particular, at frequency $f=f_{0}$, for which $\psi=\pi / 2$, i.e., when the film thickness equals half of a wavelength within the piezoelectric layer $\left(d=\lambda_{0} / 2=v / 2\right)$, one can obtain:

$$
G a=\omega \cdot C_{0} \cdot \frac{K_{e}^{2} \cdot \frac{4}{\pi} \cdot \frac{Z}{Z_{1}+Z_{2}}}{1+\left(K_{e}^{2} \cdot \frac{4}{\pi} \cdot \frac{Z}{Z_{1}+Z_{2}}\right)^{2}}
$$

This value is maximized $\left(G a=\omega C_{0} / 2\right)$ under the evident relation between the acoustic impedances of neighboring media: $Z_{1}+Z_{2}=\frac{4}{\pi} \cdot Z \cdot K_{e}^{2}$.

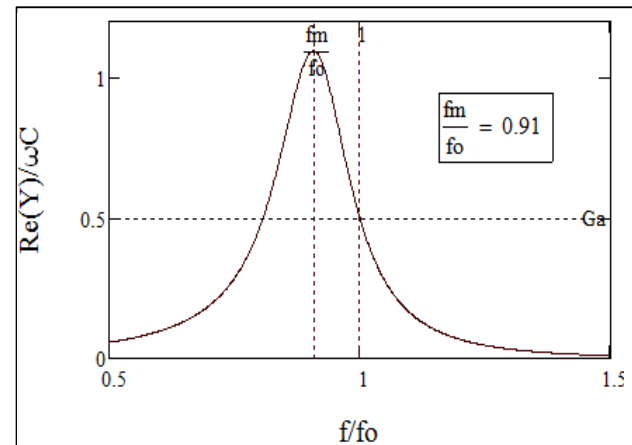

(a)

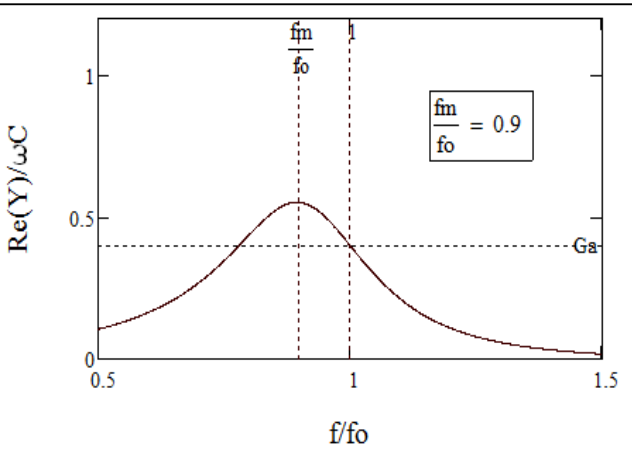

(b)

Figure 3. Frequency dependence of normalized conductance, related to a simple transducer with the AlN film under $d=\lambda_{0} / 2$, when $Z_{1}=Z_{2}=\frac{2}{\pi} \cdot K_{e}^{2} \cdot Z\left(\right.$ a), and $Z_{1}=Z_{2}=\frac{4}{\pi} \cdot K_{e}^{2} \cdot Z$ (b).

However, this is not the maximal conductance meaning over a whole spectrum (look, e.g., at Fig.3). As one can see, in order to maximize the BT's conductance at the desired frequency, one should make a piezoelectric film thinner than $\lambda_{0} / 2$. Besides, by varying $Z_{1,2}$-values one can change both magnitude and working bandwidth of the main BT's characteristic. 
Anyway, an optimization of the relation between the material parameters and thickness of piezoelectric film is needed even in this simple case. In general, a lot of input parameters (including thickness of metal electrodes) should be involved in optimization routines to improve performances of any real system. With this aim we have to apply the more rigorous modeling tools, being developed below (in Section 4).

\subsubsection{Isolated BAW resonators with infinitely thin electrodes}

One can shorten Eq. (22) notably in two particular cases, appropriate for the ideal transducer with infinitely thin electrodes, when:

1. Both edges of the piezoelectric layer to be free of external stress $\left(Z_{1,2}=0\right)(K i n o, 1987)$ :

$$
P_{33}=Y_{O O}=i \cdot \omega \cdot C_{0} \cdot\left(1-K_{e}^{2} \cdot \frac{\tan (\psi)}{\psi}\right)^{-1} ;
$$

2. One edge of the BT is free of stresses, while another border is a rigidly clamped surface $\left(Z_{1}=0 ; Z_{2} \rightarrow \infty\right)$ :

$$
P_{33}=Y_{O C}=i \cdot \omega \cdot C_{0} \cdot\left(1-K_{e}^{2} \cdot \frac{\tan (2 \psi)}{2 \psi}\right)^{-1}
$$

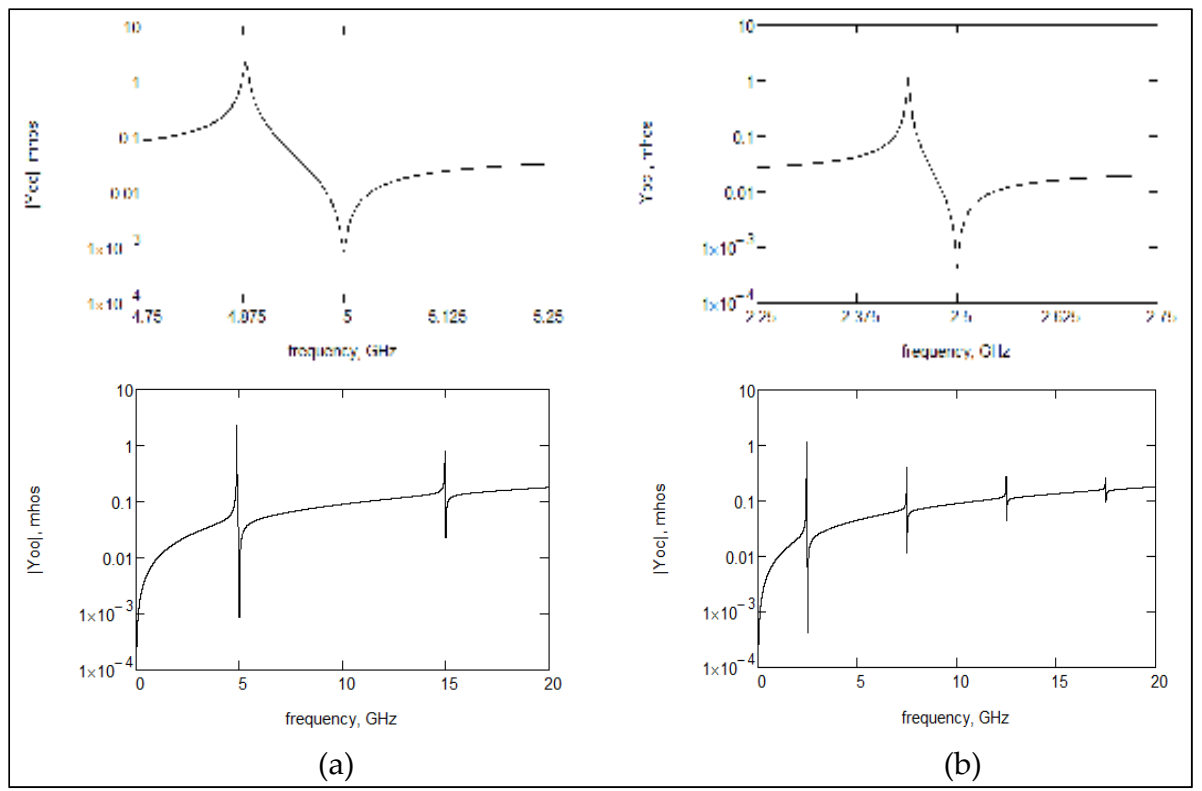

Figure 4. Admittance magnitudes of the considered idealized embodiments - $|Y o o(f)|$ (a) \& $|Y o c(f)|(\mathrm{b})$ related to BT, formed by a single aluminum nitride (AlN) film with thickness $d=1.093$ microns, calculated in a narrow (top) and wide (bottom) frequency ranges of analysis. 
The parallel resonances for the considered transducers exist under $\psi=\pi \cdot(2 n+1) / 2$, when $Y o o=0$, and if $\psi=\pi \cdot(2 n+1) / 4$, when $Y o c=0(n=0,1,2 \ldots)$.

Figures 4 illustrate functions $|Y o o(f)|$ and $|Y o c(f)|$ in logarithmic scale both for a narrow (top) and wide (bottom) frequency bandwidths of analysis, taking into account the propagation losses in AlN film, found from the Table $1\left(\zeta \approx 2.88 \cdot 10^{-3}\right.$ under $\left.f=5 \mathrm{GHz}\right)$. It should be noted that the frequency interval between the harmonic resonances in the 2nd case is twice smaller than in the first embodiment. However, the fractional ratio for $n$-th couple of resonant $\left(\left|Y\left(f_{r}\right)\right|=\max \right)$ and anti-resonant $\left(\left|Y\left(f_{a}\right)\right|=\min \right)$ frequencies satisfies the same relation for both constructions:

$$
K_{e}^{2} \cdot \tan \left(\frac{f_{n}^{(r)}}{f_{n}^{(a)}} \cdot \frac{\pi}{2}(2 n+1)\right)=\frac{f_{n}^{(r)}}{f_{n}^{(a)}} \cdot \frac{\pi}{2}(2 n+1)
$$

Besides, figures $5(\mathrm{a}, \mathrm{b})$ demonstrate the corresponding conductance frequency responses, which may be non-zero values $\left(\operatorname{Re}\left(\mathrm{Y}_{\mathrm{oo}, \mathrm{oc}}\right) \neq 0\right)$ only due to dissipation: a single reason of the power consumption within those systems, isolated hypothetically from the neighboring environment, is the transformation of acoustic energy to heat.

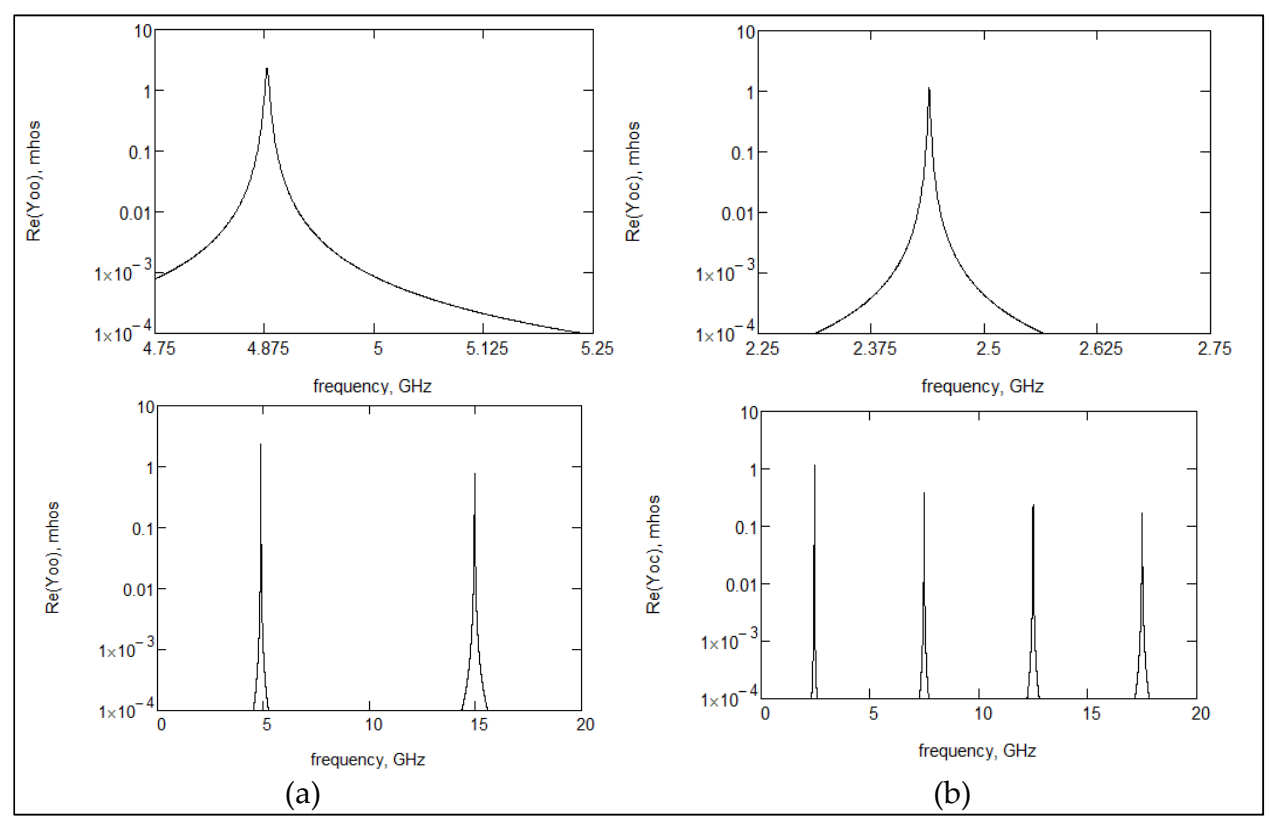

Figure 5. The conductance of considered idealized BTs, formed by a single AlN layer with thickness equaled to $\mathrm{d}=1.093$ microns and placed between infinitely thin electrodes. Calculations are made in a narrow (top) and wide (bottom) frequency ranges. 


\subsubsection{Tunable BAW reflectors containing a single piezoelectric layer}

In the recent years a demand in the electrically tunable BAW devices has revived as a result of the ability of the thin film bulk acoustic wave resonators to enable development of advanced reconfigurable/adaptable microwave circuits. In particular, the focus of the attention remained up till now on tunability which is provided when external variable bias voltage is applied to FBAR. This voltage influences the BAW velocity in the layers forming the system and therefore on its resonant frequencies (Vorobiev \& Gevorgian, 2010; Defaÿ, E. et al., 2011).

Another possibility exists to achieve the same goal on a basis of the tunable reflectors with variable electrical load, as a part of modern FBARs or SMRs.

When switch $S$ on Fig. 2 is turned to the $2^{\text {nd }}$ position, one can consider a transducer with electric load Yo, as the tunable reflector of bulk acoustic waves. Aiming to describe its operation more in detail, one should find a solution of the wave equation (8) when using a different, as compared to (13-17), set of boundary conditions.

Assuming that there is no voltage, applied to BT from an external source while looking at Fig.2, one can could conclude the following:

$$
\begin{gathered}
U_{1}+U_{2}=a_{1}+b_{1} \\
-i \cdot \tilde{c} \cdot q\left(U_{1}-U_{2}\right)-\frac{\beta}{\varepsilon} D=-i \tilde{c}_{1} \cdot q_{1} \cdot\left(a_{1}-b_{1}\right) \\
U_{1} \cdot e^{-2 i \psi}+U_{2} \cdot e^{2 i \psi}=a_{2}+b_{2} \\
-i \cdot \tilde{c} \cdot q\left(U_{1} \cdot e^{-2 i \cdot \psi}-U_{2} \cdot e^{2 i \cdot \psi}\right)-\frac{\beta}{\varepsilon} D=i \tilde{c}_{2} \cdot q_{2} \cdot\left(a_{2}-b_{2}\right) \\
D=\frac{\varepsilon}{d} V_{r}-i \beta \cdot q \cdot \frac{\sin (\psi)}{\psi} \cdot\left(U_{1} \cdot e^{-i \cdot \psi}-U_{2} \cdot e^{i \cdot \psi}\right)
\end{gathered}
$$

If $V_{\mathrm{r}}=0$ (this is so under $\left|Y_{0}\right| \rightarrow \infty$ ), then, by substituting (32) into (29) \& (31), we find a system of two coupled equations for $U_{1,2}\left(a_{1}, a_{2}\right)$ :

$$
\left\{\begin{array}{l}
\left(1+\frac{Z_{1}}{Z}-K_{e}^{2} \cdot \frac{\sin (\psi)}{\psi} \cdot e^{-i \cdot \psi}\right) \cdot U_{1}-\left(1-\frac{Z_{1}}{Z}-K_{e}^{2} \cdot \frac{\sin (\psi)}{\psi} \cdot e^{i \cdot \psi}\right) \cdot U_{2}=a_{1} \cdot \frac{2 Z_{1}}{Z} \\
\left(1-\frac{Z_{2}}{Z}-K_{e}^{2} \cdot \frac{\sin (\psi)}{\psi} \cdot e^{i \cdot \psi}\right) \cdot U_{1}-\left(1+\frac{Z_{2}}{Z}-K_{e}^{2} \cdot \frac{\sin (\psi)}{\psi} \cdot e^{-i \cdot \psi}\right) \cdot U_{2}=a_{2} \cdot \frac{2 Z_{2}}{Z}
\end{array}\right.
$$

By solving it and setting alternately two combinations of a couple $\left\{a_{1}, a_{2}\right\}(\{1,0\}$ or $\{0,1\})$, which define the fields incident on a BT, one can find all the remaining terms of $P$-matrix, mentioned above: 


$$
\begin{aligned}
& P_{11}=\frac{\left(\frac{Z_{1}-Z_{2}}{Z}\right)\left(1-K_{e}^{2} \cdot \frac{\tan (2 \psi)}{2 \psi}\right)-i \cdot \tan (2 \psi) \cdot\left(1-\frac{Z_{2} Z_{1}}{Z^{2}}-K_{e}^{2} \frac{\tan (\psi)}{\psi}\right)}{\frac{Z_{1}+Z_{2}}{Z}\left(1-K_{e}^{2} \cdot \frac{\tan (2 \psi)}{2 \psi}\right)+i \cdot \tan (2 \psi) \cdot\left(1+\frac{Z_{1} Z_{2}}{Z^{2}}-K_{e}^{2} \cdot \frac{\tan (\psi)}{\psi}\right)} \\
& P_{22}=\frac{\left(\frac{Z_{2}-Z_{1}}{Z}\right)\left(1-K_{e}^{2} \cdot \frac{\tan (2 \psi)}{2 \psi}\right)-i \cdot \tan (2 \psi) \cdot\left(1-\frac{Z_{2} Z_{1}}{Z^{2}}-K_{e}^{2} \frac{\tan (\psi)}{\psi}\right)}{\frac{Z_{1}+Z_{2}}{Z}\left(1-K_{e}^{2} \cdot \frac{\tan (2 \psi)}{2 \psi}\right)+i \cdot \tan (2 \psi) \cdot\left(1+\frac{Z_{1} Z_{2}}{Z^{2}}-K_{e}^{2} \cdot \frac{\tan (\psi)}{\psi}\right)} \\
& P_{21}=\frac{\frac{2 Z_{1}}{Z}\left(\frac{1}{\cos (2 \psi)}-K_{e}^{2} \frac{\tan (2 \psi)}{2 \psi}\right)}{\frac{Z_{1}+Z_{2}}{Z}\left(1-K_{e}^{2} \cdot \frac{\tan (2 \psi)}{2 \psi}\right)+i \cdot \tan (2 \psi) \cdot\left(1+\frac{Z_{1} Z_{2}}{Z^{2}}-K_{e}^{2} \cdot \frac{\tan (\psi)}{\psi}\right)} \\
& P_{12}=\frac{\frac{2 Z_{2}}{Z}\left(\frac{1}{\cos (2 \psi)}-K_{e}^{2} \frac{\tan (2 \psi)}{2 \psi}\right)}{\frac{Z_{1}+Z_{2}}{Z}\left(1-K_{e}^{2} \cdot \frac{\tan (2 \psi)}{2 \psi}\right)+i \cdot \tan (2 \psi) \cdot\left(1+\frac{Z_{1} Z_{2}}{Z^{2}}-K_{e}^{2} \cdot \frac{\tan (\psi)}{\psi}\right)} \\
& P_{31}=i \omega \cdot D\left(a_{1}=1 ; a_{2}=0\right)= \\
& \omega \cdot S \cdot \frac{2 \beta}{d} \cdot \frac{Z_{1}}{Z} \cdot \tan (2 \psi)\left(\frac{Z_{2}}{Z}+i \cdot \tan (\psi)\right) \\
& \overline{\frac{Z_{1}+Z_{2}}{Z}\left(1-K_{e}^{2} \cdot \frac{\tan (2 \psi)}{2 \psi}\right)+i \cdot \tan (2 \psi)\left(1+\frac{Z_{1} Z_{2}}{Z^{2}}-K_{e}^{2} \cdot \frac{\tan (\psi)}{\psi}\right)} \\
& P_{32}=i \omega \cdot D\left(a_{1}=0 ; a_{2}=1\right)= \\
& -\omega \cdot S \cdot \frac{2 \beta}{d} \cdot \frac{Z_{2}}{Z} \cdot \tan (2 \psi)\left(\frac{Z_{1}}{Z}+i \cdot \tan (\psi)\right) \\
& \overline{\frac{Z_{1}+Z_{2}}{Z}\left(1-K_{e}^{2} \cdot \frac{\tan (2 \psi)}{2 \psi}\right)+i \cdot \tan (2 \psi) \cdot\left(1+\frac{Z_{1} Z_{2}}{Z^{2}}-K_{e}^{2} \cdot \frac{\tan (\psi)}{\psi}\right)}
\end{aligned}
$$

Otherwise (if $Y_{0}^{-1} \neq 0$ ), one can express a voltage between the electrodes with the help of the Ohm's law for external electrical circuitry:

$$
i \omega \cdot D \cdot S+V_{r} \cdot Y_{0}=0
$$


As a result, Eq. (32) may be simply transformed to the relation (41):

$$
D=-i \beta \cdot q \cdot K_{Y} \cdot \frac{\sin (\psi)}{\psi} \cdot\left(U_{1} \cdot e^{-i \cdot \psi}-U_{2} \cdot e^{i \cdot \psi}\right),
$$

where $K_{Y}=\frac{Y_{o}}{Y_{o}+i \omega C_{0}}$ is the variable parameter, characterizing an electrical load.

Omitting the intermediate calculations, we present here just the resulting expressions for the modified $\tilde{P}_{11,22} \& \tilde{P}_{21,12}$ terms, of the total $P$-matrix, describing the tunable scattering (reflection and transmission) of bulk acoustic waves by the electrically loaded "one layer" tunable reflector (TR):

$$
\begin{aligned}
& \tilde{P}_{11}\left(K_{Y}\right)=\frac{\left(\frac{Z_{1}-Z_{2}}{Z}\right)\left(1-K_{e}^{2} \cdot K_{Y} \cdot \frac{\tan (2 \psi)}{2 \psi}\right)-i \cdot \tan (2 \psi)\left(1-\frac{Z_{2} Z_{1}}{Z^{2}}-K_{e}^{2} \cdot K_{Y} \cdot \frac{\tan (\psi)}{\psi}\right)}{\frac{Z_{1}+Z_{2}}{Z}\left(1-K_{e}^{2} \cdot K_{Y} \cdot \frac{\tan (2 \psi)}{2 \psi}\right)+i \cdot \tan (2 \psi)\left(1+\frac{Z_{1} Z_{2}}{Z^{2}}-K_{e}^{2} \cdot K_{Y} \cdot \frac{\tan (\psi)}{\psi}\right)} \\
& \tilde{P}_{22}\left(K_{Y}\right)=\frac{\left(\frac{Z_{2}-Z_{1}}{Z}\right)\left(1-K_{e}^{2} \cdot K_{Y} \cdot \frac{\tan (2 \psi)}{2 \psi}\right)-i \cdot \tan (2 \psi)\left(1-\frac{Z_{2} Z_{1}}{Z^{2}}-K_{e}^{2} \cdot K_{Y} \cdot \frac{\tan (\psi)}{\psi}\right)}{\frac{Z_{1}+Z_{2}}{Z}\left(1-K_{e}^{2} \cdot K_{Y} \cdot \frac{\tan (2 \psi)}{2 \psi}\right)+i \cdot \tan (2 \psi)\left(1+\frac{Z_{1} Z_{2}}{Z^{2}}-K_{e}^{2} \cdot K_{Y} \cdot \frac{\tan (\psi)}{\psi}\right)} \\
& \tilde{P}_{21}\left(K_{Y}\right)=\frac{\frac{2 Z_{1}}{Z}\left(\frac{1}{\cos (2 \psi)}-K_{e}^{2} \cdot K_{Y} \frac{\tan (2 \psi)}{2 \psi}\right)}{\frac{Z_{1}+Z_{2}}{Z}\left(1-K_{e}^{2} \cdot K_{Y} \cdot \frac{\tan (2 \psi)}{2 \psi}\right)+i \cdot \tan (2 \psi)\left(1+\frac{Z_{1} Z_{2}}{Z^{2}}-K_{e}^{2} \cdot K_{Y} \cdot \frac{\tan (\psi)}{\psi}\right)} \\
& \tilde{P}_{12}\left(K_{Y}\right)=\frac{\frac{2 Z_{2}}{Z}\left(\frac{1}{\cos (2 \psi)}-K_{e}^{2} \cdot K_{Y} \frac{\tan (2 \psi)}{2 \psi}\right)}{\frac{Z_{1}+Z_{2}}{Z}\left(1-K_{e}^{2} \cdot K_{Y} \cdot \frac{\tan (2 \psi)}{2 \psi}\right)+i \cdot \tan (2 \psi)\left(1+\frac{Z_{1} Z_{2}}{Z^{2}}-K_{e}^{2} \cdot K_{Y} \cdot \frac{\tan (\psi)}{\psi}\right)}
\end{aligned}
$$

Assuming that reactance is used as the electrical load (either capacitor $\tilde{C}$ or inductor $\tilde{L}$ ), one can take into consideration also the finite resistance $\left(R_{\mathrm{e}}\right)$ of TR's electrodes, placing formally - in parallel to TR a shunting conductance $G_{S} \approx \omega_{0}^{2} \cdot C_{0}^{2} \cdot R_{e}=\omega_{0} \cdot C_{0} / Q_{0}$ :

$$
Y_{0}=\left\{\begin{array}{l}
i \cdot \omega \cdot \tilde{C}\left(1-\frac{i}{Q_{C}}\right)+\frac{\omega_{0} \cdot C_{0}}{Q_{0}} \Leftrightarrow \text { for capacitive load; } \\
\frac{1}{i \cdot \omega \cdot \tilde{L}}\left(1+\frac{i}{Q_{L}}\right)+\frac{\omega_{0} \cdot C_{0}}{Q_{0}} \Leftrightarrow \text { for inductive load, }
\end{array}\right.
$$


where $Q_{\mathrm{C}}$ - \& $Q_{\mathrm{L}}$ - values mean the load's $Q$-factor (being different, generally, for capacitors and inductors).

It is convenient to characterize $Y_{0}$-value by the dimensionless parameter ${ }^{4}$ :

$$
\delta=-\frac{\operatorname{Im}\left(Y_{0}\right)+\omega_{0} \cdot C_{0}}{\omega_{0} \cdot C_{0}}
$$

Thus, the "tuning" parameter $K_{Y}$, introduced above, may be represented in the following form:

$$
\begin{gathered}
K_{Y}=\frac{y_{0}}{y_{0}+f / f_{0}}, \text { where } \\
y_{0}=\left\{\begin{array}{l}
-(1+\delta) \frac{f}{f_{0}} \cdot\left(1-\frac{i}{Q_{C}}\right)-\frac{i}{Q_{0}}, \quad \text { if } \quad \delta \leq-1 \text { (for capacitive load) } \\
-(1+\delta) \cdot \frac{f_{0}}{f} \cdot\left(1+\frac{i}{Q_{L}} \cdot \frac{f_{0}}{f}\right)-\frac{i}{Q_{0}}, \quad \text { otherwise (for inductive load) }
\end{array}\right.
\end{gathered}
$$

Here Qc \& QL mean the Q-factors of capacitive and inductive loads, respectively, while $f_{0}=$ $\omega_{0} / 2 \pi$ is that frequency, at which external inductance compensates the BT's static capacitance $(\delta=0)$. Figures $(6-8)$ show some examples illustrating the scattering features of TR in the considered simplified variant when using aluminum nitride (AIN) piezoelectric film, supporting the longitudinal bulk wave mode.

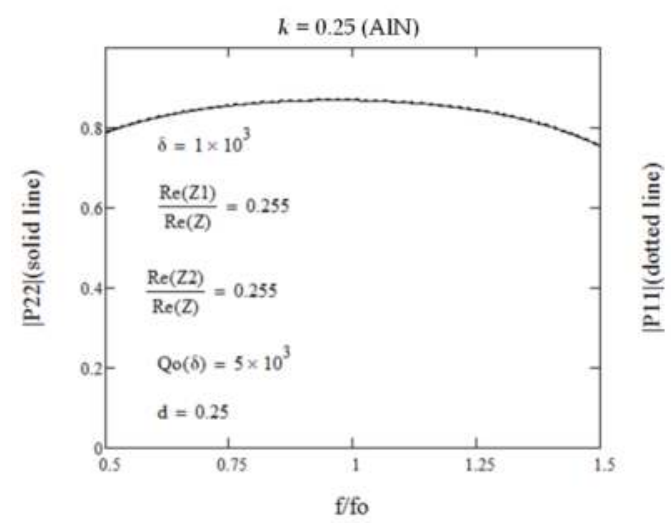

Figure 6. Reflectivity of the short-circuited BT ( $\delta \rightarrow \infty \Leftrightarrow \mathrm{KY}=1)$ with the quarter-wavelength thickness of AlN film $\left(d=\lambda_{0} / 4=v / 2 f_{0}\right)$.

Contrary to the short-circuited BT (Fig.6), the reflectivity may practically vanish under properly found (for a chosen frequency) load's reactance, if the load's Q-factor is enough high (Fig.7b).

${ }^{4} \delta=-1 \& \delta=0$ mean the open- \& short-circuited modes, correspondingly. 


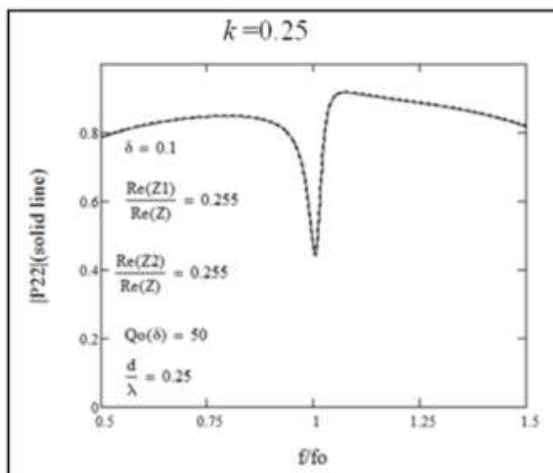

a)

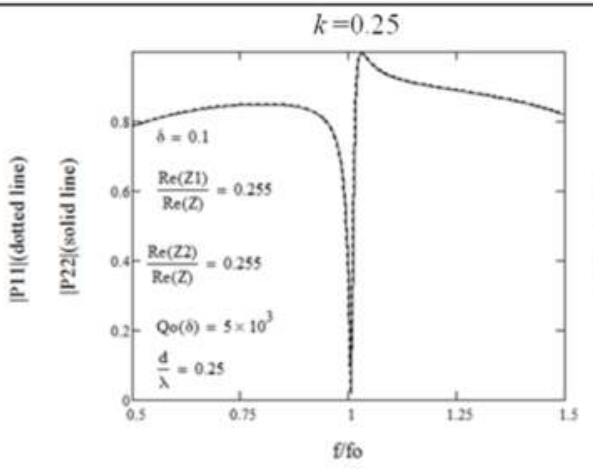

b)

Figure 7. Antireflecting effect under different values of the load'd Q-factor.

Physical sense of the mentioned tunability is quite evident: a current, induced because of inverse piezoelectricity by incident waves on electrodes, provides "secondary" voltage over the load, generating - in turn - the waves in the acoustic channel (which propagate, generally, in both opposite directions).

The re-radiated waves interfere with the reflected and transmitted ones, which appear in the inhomogeneous elastic channel under electrical shorting $\left(Y_{0} \rightarrow \infty\right)$. The load' change results, surely, in the amplitude and phase variation of the reflected and passed through BT waves.

The antireflecting properties of a similar structure may appear when a wave, regenerated backward, comes into antiphase with the "elastic" reflections, cancelling the resulting backward wave almost at all. The finite $Q$-factor of a load results in a several degradation of the antireflecting effect (Fig.7b).

\subsubsection{Energy balance and the second law of thermodynamics as checking points}

There are two conditions here, which allow us to verify the modeling adequacy bearing in mind that the power flow, carried by acoustic wave with amplitude $U$ in the nonpiezoelectric medium, equals $i \omega \cdot U^{*} \cdot T=\omega \cdot \operatorname{Re}(c \cdot q) \cdot|U|^{2}=\omega^{2} \cdot \operatorname{Re}(Z) \cdot|U|^{2}$ (Auld, 1973).

First of all, in the absence of the beams coming from the outside $\left(a_{1}=a_{2}=0\right)$, the total power of acoustic waves, radiated by a transducer under applied voltage $V$, is equal to

$$
P_{T}=\left|V^{2}\right| \cdot \omega^{2} \cdot S \cdot\left\{\operatorname{Re}\left(Z_{2}\right) \cdot\left|P_{23}\right|^{2}+\operatorname{Re}\left(Z_{1}\right) \cdot\left|P_{13}\right|^{2}\right\}
$$

Accordingly, we should always check whether the energy balance condition is satisfied requiring the following obligatory relation between $P_{T}$-value and the transducer conductance, indicating the total power consumed from electrical source:

$$
P_{T} \leq \operatorname{Re}\left(P_{33}\right) \cdot\left|V^{2}\right|
$$


Equality in (51) holds exactly only in the lossless case: in general, a part of acoustic power transforms to heat within BT's body and can't be radiated outside it.

On the other hand, in perfect accordance with the 2nd law of thermodynamics, the total part of acoustic power, passed through the reflector, cannot depend on the propagation direction of the incident wave (Fig.8a) ${ }^{5}$. This condition should always be satisfied, even in the presence of dissipation in asymmetrical case (if $Z_{1} \neq Z_{2}$ ), when a layer may have different reflectivity with respect to the waves launching upon it from opposite directions (Fig.8b):

$$
\begin{gathered}
\left|\tilde{P}_{21}\right|^{2} \cdot \operatorname{Re}\left(Z_{2}\right) / \operatorname{Re}\left(Z_{1}\right)=\left|\tilde{P}_{12}\right|^{2} \cdot \operatorname{Re}\left(Z_{1}\right) / \operatorname{Re}\left(Z_{2}\right), \text { that is } \\
\left|\tilde{P}_{21}\right| \cdot \operatorname{Re}\left(Z_{2}\right)=\left|\tilde{P}_{12}\right| \cdot \operatorname{Re}\left(Z_{1}\right)
\end{gathered}
$$

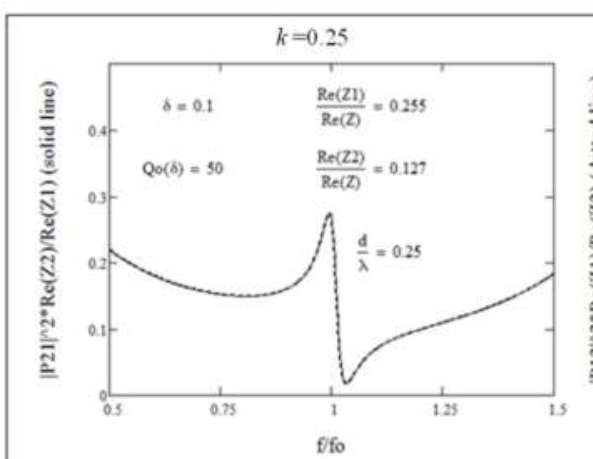

a)

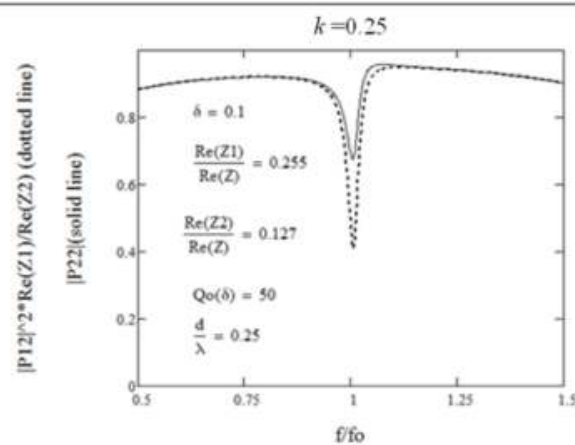

b)

Figure 8. The peculiarity of the BAW scattering by asymmetrical tunable reflector $\left(Z_{1} \neq Z_{2}\right)$.

As calculations show, Eqs. (21-22) and (42-45) agree absolutely with the conditions (51-52).

\subsubsection{Tunability of the "frontal" BAW reflectors}

The tuning possibilities of acoustoetectric BAW transducers as reflectors have been investigated first long ago (Gristchenko, 1975). It was proved that one could have control over the reflected and transmitted acoustic power by means of variable reactance, connected to BT's electrodes.

Note that a physically analogous phenomenon, concerning the tunable scattering of surface acoustic waves (SAWs), has been widely investigated even earlier than tunability of BAW devices. Smith et al. (1969) first have presented an analysis of the interdigital transducer (IDT) basing on the equivalent circuit model in the absence of distributed feedback (DFB) caused by SAW reflections from electrodes as the periodic inhomogeneities. The corresponding expression for the regenerative reflection coefficient of IDT (with the total

${ }^{5}$ Otherwise, a dissipative half-space from a one side of reflector should be heated as compared with the neighbor halfspace, disturbing the condition of thermodynamic equilibrium. 
admittance $Y$ ) was obtained. It can be expressed, within the constant phase multiplier, in the following simple form:

$$
R=\frac{\operatorname{Re}(Y)}{Y+Y_{0}}
$$

Later Sandler and Sveshnikov (1981), basing on the Coupling-of-Modes (COM) analysis, have developed the more general model for IDT, taking into account distributed feedback also. As they have shown theoretically and, in co-author with Paskhin, experimentally, if a $Q$-factor of a reactance is high enough, then the reflection coefficient of the interdigital reflector may be electrically varied practically from zero to unity for any efficacy of Bragg's reflections. This circumstance was used to manufacture the tunable SAW resonators, as well as to suppress electrically the triple-transit signals in ordinary bandpass SAW filters (Paskhin et al., 1981).

Then, the phase-shifting features of a reflector, manufactured as a single phase unidirectional transducer (SPUDT) with variable electrical load, has been discovered (Sveshnikov \& Filinov, 1988). It was revealed that the phase value of the SPUDT reflection coefficient can be varied electrically over an interval $[0 ; 2 \pi]$ at the stopband frequency, if the SAW beam is launched upon a SPUDT from the direction of its predominant radiation. It means that by changing variable reactance $Y_{0}$ one can fluently change resonant frequency of the resonator, containing similar reflector, over a frequency interval $\Delta f$ between its neighboring resonant frequencies.

Due to the physical propinquity of SPUDT with the frontal BT (which has a unidirectional nature in principle), it became clear that the same phenomenon has to appear for the frontal BAW transducer, placed on the crystal surface and used as the "one-side " tunable mirror. This effect was well founded further both by simplified analytical model (Sveshnikov, 1995), and by numerical calculations made for multimode BAW resonators (Kucheryavaya, et al., 1995).

Indeed, assuming that there is the air above the boundary $\mathrm{x}=0$ on Fig.2 (when a shift $\mathrm{S}$ there is in the second position) and neglecting a dissipation in the middle layer, a surface $x=0$ should be considered as the perfect elastic mirror: $\left|\frac{U_{2}}{U_{1}}\right|$ practically equa1s unity ${ }^{6}$.

Owing to system's linearity, the total reflection coefficient of the loaded BT $\left(R=b_{2} / a_{2}\right)$, being a superposition of the elastic and regenerative terms, within the inessential phase constant may be represented as follows:

$$
\begin{gathered}
R=1+\frac{A \cdot \operatorname{Re}(Y)}{Y+Y_{0}}=1+\frac{A}{1+\alpha+i \cdot \theta}, \text { where } \\
\alpha=\frac{\operatorname{Re}\left(Y_{0}\right)}{\operatorname{Re}(Y)} ; \quad \theta=\frac{\operatorname{Im}\left(Y+Y_{0}\right)}{\operatorname{Re}(Y)},
\end{gathered}
$$

\footnotetext{
${ }^{6}$ Because, for example, $Z_{\text {air }} / Z_{\mathrm{AlN}} \sim 10^{-5}$.
} 
while $A$ is unknown constant coefficient. As it doesn't depend on $Y_{0}$-value, one can find it assuming for the nonce a load to be the lossless reactance $(\alpha=0)$. In this case, due to the energy balance, an equality $|\mathrm{R}|=1$ holds too. The last relation may be satisfied for arbitrary $\theta$ -value only under $\mathrm{A}=-2$. Thus, we get the following simple formula, being valid even in the presence of Ohm loss in electrical load $(\alpha \neq 0)$, neglecting only the BAW attenuation in piezoelectric film (Sveshnikov, 1995):

$$
R=\frac{1-\alpha-i \theta}{1+\alpha+i \theta}=|R| \cdot e^{i \Phi_{R}}, \text { where } \quad \Phi_{R}=-a \tan \left(\frac{\theta}{1-\alpha}\right)-a \tan \left(\frac{\theta}{1+\alpha}\right)
$$

As a consequence of the reciprocity principle, under the electrical matching ( $\alpha=1$ and $\theta=0$ ) the mentioned reflectivity should disappear $(R=0)$, because of the perfect unidirectionality of this frontal BT (all the incident power is absorbed in a load).

Taking into account (21) \& (54), by numerical calculations made under condition $Z_{1}=0$ one can always ascertain that Eq.(43), obtained rigorously, absolutely coincides with (55) in the absence of layer's viscosity ${ }^{7}$. For example, if $\psi=\pi / 2$, then $\tan (2 \psi) \cdot \tan (\psi)=-2$, and

$$
\tilde{P}_{22}=\frac{1-i \cdot \frac{4}{\pi} \cdot \frac{Z}{Z_{2}} K_{e}^{2} \cdot K_{Y}}{1+i \cdot \frac{4}{\pi} \cdot \frac{Z}{Z_{2}} K_{e}^{2} \cdot K_{Y}}
$$

It is clear from (55) also that under condition of enough small Ohm losses $(\alpha<1)$, if the reactive load is widely varied, from a very large capacitance $(\theta \rightarrow \infty)$ to a very small inductance $(\theta \rightarrow-\infty)$, then the reflection phase is changed all around a circle: $-\pi<\Phi_{R}<\pi$. Nonetheless, under the finite $\mathrm{Q}$ - factor of a load, $|R|$-value may decrease notably during the tuning process. Figures $9 a \& 9 b$ illustrate this effect in two cases: a) under real $Q$-factors of electrical circuitry, and b) when these $Q$-factors assumed to be ten times larger $\left(f_{0}=\right.$ 2GHz).

The larger ratio $\mathrm{G}=\operatorname{Re}\left(P_{33}\right) / \omega C_{0}$ between the transducer conductance and its "static" susceptance, the smaller the mentioned falling of TR's reflectivity. As it is clear from (56) one can increase G-value by decreasing the ratio $\left|Z_{2} / Z\right|$. However, on this way we have not enough variety of the impedance combinations, when using real materials.

Another technological possibility must be analyzed also to improve the functional features of tunable BAW reflectors. It concerns manufacturing of the multi-layered BAW transducer, containing a number $\left(N_{p}\right)$ piezoelectric layers placed between electrodes with alternating polarity - similarly to the interdigital transducer (IDT) of surface acoustic waves. It was clear that G-value in this case may rise almost linearly with increasing $N_{\mathrm{p}}$. On the other hand, the wave propagation within the extended (in the longitudinal direction) reflector will bring us

\footnotetext{
${ }^{7}$ This fact is another confirmation of the simulation validity.
} 
additional losses too. So, it is necessary to create a proper model, to be able optimize the parameters of actual BAW devices in the presence of unavoidable dissipation.

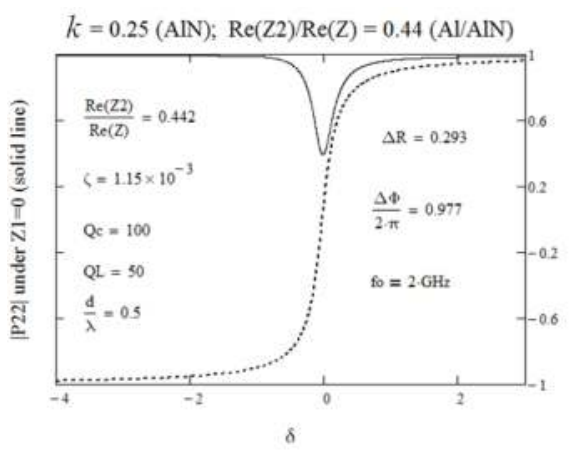

a)

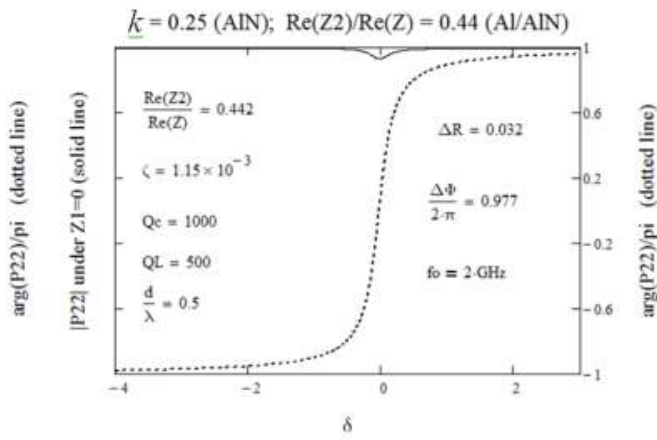

b)

Figure 9. Tunability of the frontal BAW reflector by varying a load's reactance: an interval $\Delta \Phi$ of variation of the reflection coefficient phase $\Phi=\arg \left(P 22\left(f_{0}\right)\right)$ is close to $2 \pi$ when $\delta \in[-4 ;+3]$.

\section{P-matrix of BAW transducer with multiple electrodes}

Suppose, there is a sequence of piezoelectric layers, placed between metal electrodes with alternating polarity, which form a multi-layer BAW transducer (MBT), in analogy with IDT (Fig.10).

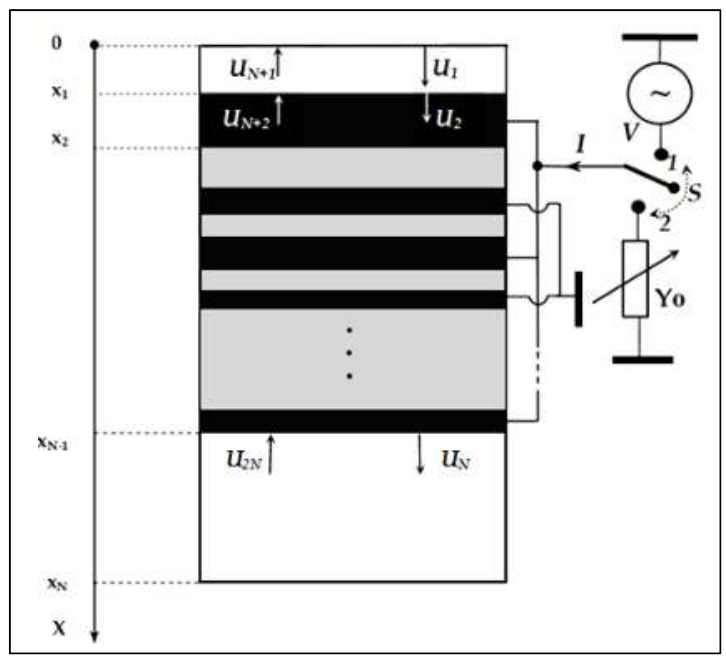

Figure 10. Schematic representation of multi-layer transducer of bulk acoustic waves, which becomes tunable reflector, when being loaded by variable reactance.

The $P$-matrix of an BT, containing a number of arbitrary layers in the amount of $N_{\mathrm{t}}=2 N_{\mathrm{p}}+1$ (including electrodes), depends on materials of the neighboring media of acoustic track, 
where a transducer should be placed. So, when finding the BT's $P$-matrix we have to involve into consideration also a pair of the frontier non-piezoelectric layers, made from the arbitrary materials. The total number of layers, to be taken into account at this point, equals $N=N_{\mathrm{t}}+2=2 N_{\mathrm{p}}+3$.

Denoting $d_{j}=x_{j}-x_{j-1}$ and following the above-mentioned numeration of acoustic waves, appeared inside a system either because of applied voltage $(V \neq 0)$, or due to external beams under short-circuiting condition $(V=0)$, we consider the boundary conditions (9-10) at the interfaces $x_{1}, x_{2} \ldots x_{\mathrm{N}-1}$, bearing in mind (in analogy with (17)) that within $j$-th film

$$
D_{j}=\frac{\varepsilon_{j}}{d_{j}} \delta V_{j}-i \beta_{j} \cdot q_{j} \cdot \frac{\sin \left(\psi_{j}\right)}{\psi_{j}} \cdot\left(U_{j} \cdot e^{-i \cdot \psi_{j}}-U_{j+N} \cdot e^{i \cdot \psi_{j}}\right),
$$

where $\delta V_{j}=V_{j-1}-V_{j}$ mean the voltage drop upon $j$-th layer.

Besides, there are two boundary conditions at the borders $x=x_{0}=0 \& x=x_{N}$. One can characterize them by two parameters $B_{1,2}$, which may accept three meanings: 1$) B_{1,2}=1$, relating to free edges $\left.\left(T\left(x_{0, N}\right)=0\right) ; 2\right) B_{1,2}=-1$, relating to the rigidly clamped borders $\left(u\left(x_{0, N}\right)\right.$ $=0)$, and 3) $B_{1,2}=0$, imitating the perfect matching of transducer with neighboring acoustic channels (when there are no waves, coming to transducer from outside):

$$
\left\{\begin{array}{l}
U_{1}=B_{1} \cdot U_{N+1} \\
U_{2 N}=B_{2} \cdot e^{-i \cdot 2 q_{N} \cdot d_{N}} \cdot U_{N}
\end{array}\right.
$$

So, one can get a linear system of $2 N$ coupled equations for amplitudes $U_{m}(m=1 \ldots 2 N)$, which may be written in the following matrix form for two vectors, characterizing the spatial distribution of acoustic fields $\left(\vec{U}^{(V)} \& \vec{U}^{(a)}\right)$, induced within a device either by applied voltage, or by external beams incident on a short circuited transducer from outside:

$$
\vec{U}^{(V, a)}=\hat{M} \cdot \vec{U}^{(V, a)}+\vec{H}^{(V, a)}
$$

At this time the vectors $\vec{H}^{(V)} \& \vec{H}^{(a)}$ define in (59) the sources of the BAW excitation, appeared both because of the applied voltage $V$ (when $a_{1}=a_{2}=0$ ) and due to external acoustic fields with unit amplitudes, launching on BT at the cross-sections $x=0\left(a_{1}=1, a_{2}=0\right)$ or $x=\mathrm{xN}_{\mathrm{N}}\left(a_{1}=0, a_{2}=1\right)$ :

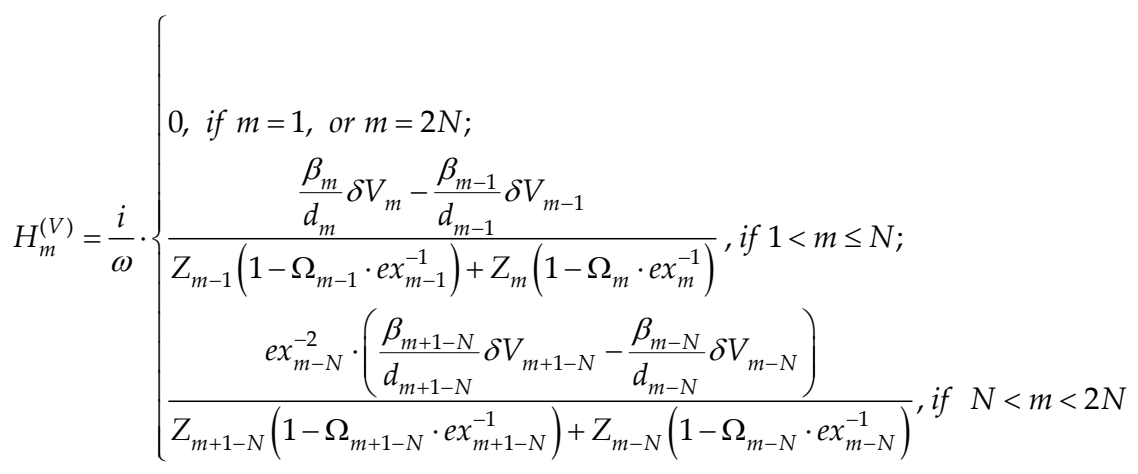




$$
H_{m}^{(a)}=a_{1} \cdot \delta(m, 1)+a_{2} \cdot \delta(m, 2 N)
$$

where $e x_{j}=\exp \left(i \cdot \psi_{j}\right) ; \Omega_{j}=\frac{k_{j}^{2}}{1+k_{j}^{2}} \cdot \frac{\sin \left(\psi_{j}\right)}{\psi_{j}} ; a_{1,2}=1$ or 0 , and $\delta(m, n)$ is the Kronecker symbol $(m, n=1 \ldots 2 N)$.

The "local “ scattering matrix in (59) $\hat{M}=\left(\begin{array}{ll}\hat{M} 11 & \hat{M} 12 \\ \hat{M} 21 & \hat{M} 22\end{array}\right)$, characterizing the interaction of counter-propagating waves at all interfaces, is formed by four $(N \times N)$ sub-matrices:

$$
\begin{aligned}
& \hat{M} 11=\left(\begin{array}{cccccc}
0 & 0 & \cdots & 0 & 0 & 0 \\
t_{1}^{(+)} \cdot e x_{1}^{-2} & 0 & \cdots & 0 & 0 & 0 \\
0 & t_{2}^{(+)} \cdot e x_{2}^{-2} & \cdots & 0 & \vdots & 0 \\
0 & 0 & \ddots & \vdots & 0 & \vdots \\
\vdots & \vdots & \vdots & t_{N-2}^{(+)} \cdot e x_{N-2}^{-2} & 0 & 0 \\
0 & 0 & \cdots & 0 & t_{N-1}^{(+)} \cdot e x_{N-1}^{-2} & 0
\end{array}\right) ; \\
& \hat{M} 22=\left(\begin{array}{cccccc}
0 & t_{1}^{(-)} \cdot e x_{1}^{-2} & 0 & \ldots & 0 & 0 \\
0 & 0 & t_{2}^{(-)} \cdot e x_{2}^{-2} & \ldots & 0 & 0 \\
0 & 0 & 0 & \ldots & \vdots & 0 \\
0 & 0 & \vdots & \ddots & t_{N-2}^{(-)} \cdot e x_{N-2}^{-2} & \vdots \\
\vdots & \vdots & 0 & \ldots & 0 & t_{N-1}^{(-)} \cdot e x_{N-1}^{-2} \\
0 & 0 & 0 & \ldots & 0 & 0
\end{array}\right) ; \\
& \hat{M} 12=\left(\begin{array}{cccccc}
B_{1} & 0 & 0 & \cdots & 0 & 0 \\
0 & r_{1}^{(+)} & 0 & \cdots & 0 & 0 \\
0 & 0 & r_{2}^{(+)} & \cdots & \vdots & 0 \\
0 & 0 & 0 & \ddots & 0 & \vdots \\
\vdots & \vdots & 0 & \cdots & r_{\mathrm{N}-2}^{(+)} & 0 \\
0 & 0 & 0 & \cdots & 0 & r_{\mathrm{N}-1}^{(+)}
\end{array}\right) \\
& \hat{M} 21=\left(\begin{array}{cccccc}
r_{1}^{(-)} e x_{1}^{-4} & 0 & 0 & \cdots & 0 & 0 \\
0 & r_{2}^{(-)} e x_{2}^{-4} & 0 & \cdots & 0 & 0 \\
0 & 0 & r_{3}^{(-)} e x_{3}^{-4} & \cdots & \vdots & 0 \\
0 & 0 & 0 & \ddots & 0 & \vdots \\
\vdots & \vdots & 0 & \cdots & r_{N-1}^{(-)} e x_{N-1}^{-4} & 0 \\
0 & 0 & 0 & \cdots & 0 & B_{2} \cdot e x_{N}^{-4}
\end{array}\right)
\end{aligned}
$$

Here $t_{j}^{(+)} \& t_{j}^{(-)}$are the transmission coefficients of BAW beams through $j$-th interface for waves, propagating under $\delta V_{j}=0$ forward (in ", $+\mathrm{x}^{\prime}$ direction) \& backward (in "- $\mathrm{x}^{\prime \prime}$ direction), respectively; $r_{j}^{(+)} \& r_{j}^{(-)}$have a sense of reflection coefficients, describing (under $\delta V_{j}=0$ ) 
reflection at $j$-th interface of waves, propagating after reflection in ", $+x^{\prime \prime} \&$ "- $x^{\prime \prime}$ directions, correspondingly:

$$
\begin{aligned}
& t_{j}^{(+)}=\frac{2 \cdot\left(1-\Omega_{j} \cdot \cos \left(\psi_{j}\right)\right) \cdot Z_{j}}{Z_{j+1}\left(1-\Omega_{j+1} \cdot e x_{j+1}^{-1}\right)+Z_{j}\left(1-\Omega_{j} \cdot e x_{j}^{-1}\right)} ; \\
& t_{j}^{(-)}=\frac{2 \cdot\left(1-\Omega_{j+1} \cdot \cos \left(\psi_{j+1}\right)\right) \cdot Z_{j+1}}{Z_{j+1}\left(1-\Omega_{j+1} \cdot e x_{j+1}^{-1}\right)+Z_{j}\left(1-\Omega_{j} \cdot e x_{j}^{-1}\right)} ; \\
& r_{j}^{(+)}=\frac{Z_{j+1}\left(1-\Omega_{j+1} \cdot e x_{j+1}\right)-Z_{j}\left(1-\Omega_{j} \cdot e x_{j}^{-1}\right)}{Z_{j+1}\left(1-\Omega_{j+1} \cdot e x_{j+1}^{-1}\right)+Z_{j}\left(1-\Omega_{j} \cdot e x_{j}^{-1}\right)} ; \\
& r_{j}^{(-)}=\frac{Z_{j}\left(1-\Omega_{j} \cdot e x_{j}\right)-Z_{j}\left(1-\Omega_{j+1} \cdot e x_{j+1}^{-1}\right)}{Z_{j+1}\left(1-\Omega_{j+1} \cdot e x_{j+1}^{-1}\right)+Z_{j}\left(1-\Omega_{j} \cdot e x_{j}^{-1}\right)},
\end{aligned}
$$

where $Z_{j}$ means complex acoustic impedance of $j$-th layer (see (19a)).

The following evident equality solves (59) when introducing the $(2 N \times 2 N)$ unit matrix $\hat{I}$ :

$$
\vec{U}^{(V, a)}=(\hat{I}-\hat{M})^{-1} \cdot \vec{H}^{(V, a)}
$$

In accordance with (62) the spatial distribution of electric displacement $D_{j}$, expressed using (57) through the spatial distribution $U_{j} \& U_{j+N}$, may be also represented as a superposition of two terms induced either by the voltage or by the external acoustic beams: $D_{j}=D_{j}^{(V)}+D_{j}^{(a)}$.

Thus, when using (62), one could simply obtain all terms of the desired $P$-matrix related to arbitrary multi-layer transducer:

$$
\begin{gathered}
P_{11}=\left.e x_{1}^{2} \cdot U_{N+1}^{(a)}\right|_{a_{1}=1 ; a_{2}=0} ; \quad P_{12}=\left.U_{N+1}^{(a)}\right|_{a_{1}=0 ; a_{2}=1} ; P_{13}=e x_{1}^{2} \cdot U_{N+1}^{(V)} / V \\
P_{21}=\left.U_{N}^{(a)}\right|_{a_{1}=1 ; a_{2}=0} ; \quad P_{22}=\left.U_{N}^{(a)}\right|_{a_{1}=0 ; a_{2}=1} ; \quad P_{23}=U_{N}^{(V)} / V \\
P_{31}=\left.\frac{i \omega \cdot S}{V} \cdot \sum_{j} D_{j}^{(a)}\right|_{a_{1}=1 ; a_{2}=0} ; \quad P_{32}=\left.\frac{i \omega \cdot S}{V} \cdot \sum_{j} D_{j}^{(a)}\right|_{a_{1}=0 ; a_{2}=1} ; P_{33}=\frac{i \omega \cdot S}{V} \cdot \sum_{j} D_{j}^{(V)} \cdot \delta V_{j}
\end{gathered}
$$

Note, the equalities (63-65) concern the same $P$-parameters as (20-21) \& (34-39), derived above for the "one-layer" BT. In order to obtain the scattering parameters, characterizing tunable multi-layer reflector, loaded by variable electrical admittance $Y_{0}$, it is convenient to apply, using (63-65), the well-known relations (see, e.g., Hashimoto, 2009):

$$
\tilde{P}_{11,22}=P_{11,22}-\frac{P_{31,32} \cdot P_{13,23}}{P_{33}+Y_{0}} ; \quad \tilde{P}_{21,12}=P_{21,12}-\frac{P_{32,31} \cdot P_{23,13}}{P_{33}+Y_{0}}
$$


Naturally, the found relations (63-66) for generalized $P$-matrix meet both of the fundamental requirements (51-52), confirming the simulation validity.

Figs.11 illustrate some frequency responses, representing conductance of the multi-layered frontal transducer $\left(B_{1}=1\right)$, which contains AlN films with thickness $d_{p}=v_{p} /\left(2 f_{0}\right)$, placed between infinitely thin, though infinitely conductive, $A l$ electrodes. The curves, found under the assumption that the "bottom" layer of transducer is perfectly matched with the adjacent acoustic channel $\left(B_{2}=0\right)$, was normalized by the product of $N p$-value on a conductance, calculated at frequency $f_{0}$ for the "one layer" BT ${ }^{8}$.

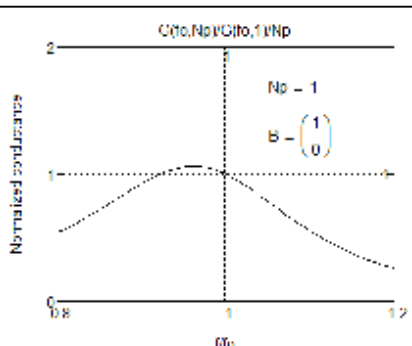

(a)

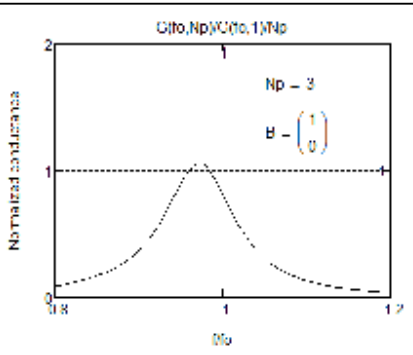

(b)

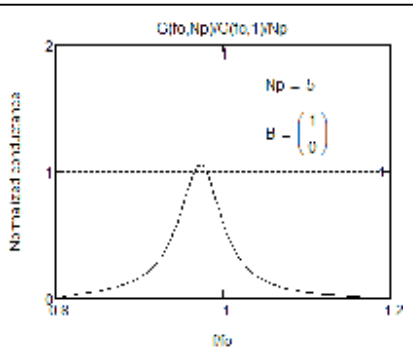

(c)

Figure 11. The normalized conductance of BTs, containing different numbers $(N p)$ of AlN layers with thickness $d=v /\left(2 f_{0}\right)$, placed between infinitely thin, though perfectly conductive $A l$ electrodes, under a different number of piezoelectric layers: $N p=1$ (a), 3 (b) and 5 (c).

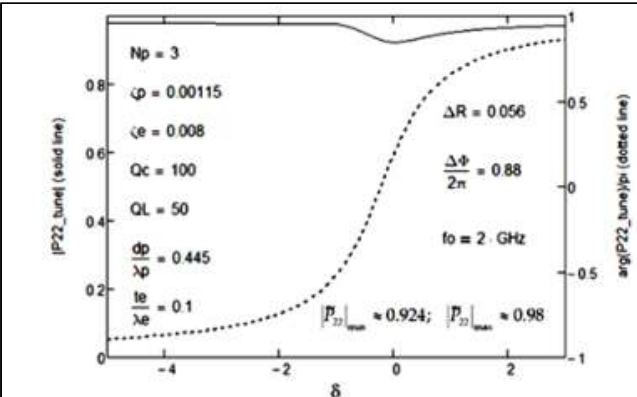

(a)

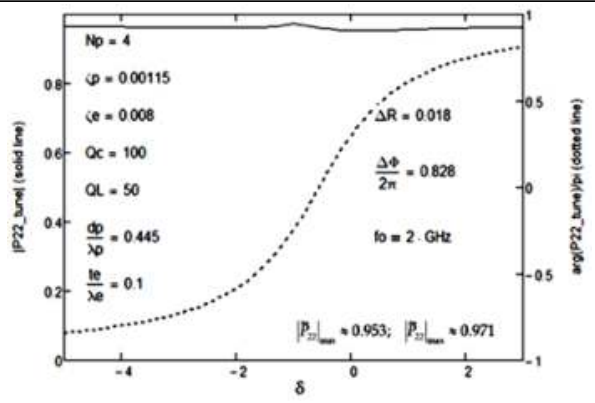

(b)

Figure 12. Tuning of multi-layered frontal TR, containing three (b) \& four (c) piezoelectric films with thickness $d_{\mathrm{p}}=0.445 \cdot \lambda_{\mathrm{p}}$, when $\delta \in[-4 ;+3] \& f=f_{0}=v_{p} / \lambda_{p}=2 \mathrm{GHz}$ (electric resistance of $A l$ electrodes with thickness $t_{\mathrm{e}}=0.1 \cdot \lambda_{\mathrm{e}}$ assumed to be negligible yet).

As it is clear from Figs.12 one can minimize the undesired variation of TR's reflectivity, occurring during the tuning process, by using multilayer structures (compare with Fig.9a). However, this is done at the expense of certain reduction of the reflection coefficient magnitude in consequence of the viscous damping in TR with increased longitudinal size.

${ }^{8}$ If $N p=1$, these results numerically coincide with the analytical ones, found above in Section 3.1.2. 
We don't pay attention here to a separate task, concerning optimization of the system parameters, as its solution depends on concrete specifications and technological capabilities of the BAW device manufacturing. However, the developed model is just the well suited instrument facilitating a solution of the multi-parametric optimization problem, bearing in mind the finite thickness of electrodes as well.

\section{Tunable SMR}

As an example of universal modeling, we'd like to demonstrate, how one can calculate characteristics of solidly mounted resonators (SMR), containing, in particular, the above mentioned multi-layered tunable reflector.

Let us consider, for instance, a device, consisting of two parts: 1) frontal TR, placed in the domain $x<0$ including $N p$ piezoelectric films placed between $A l$ electrodes, and 2) "twoport" domain, with input \& output transducers (containing $N P_{1} \& N P_{2}$ piezoelectric films), mounted on the bottom substrate by using an intermediate acoustic Bragg reflector (BR). BR consists of alternating high and low acoustic impedance layers (e.g., Mo and $\mathrm{SiO}_{2}$ ), in amount $N r$, manufactured in order to select only one acoustic resonance in this potentially multi-resonant system.

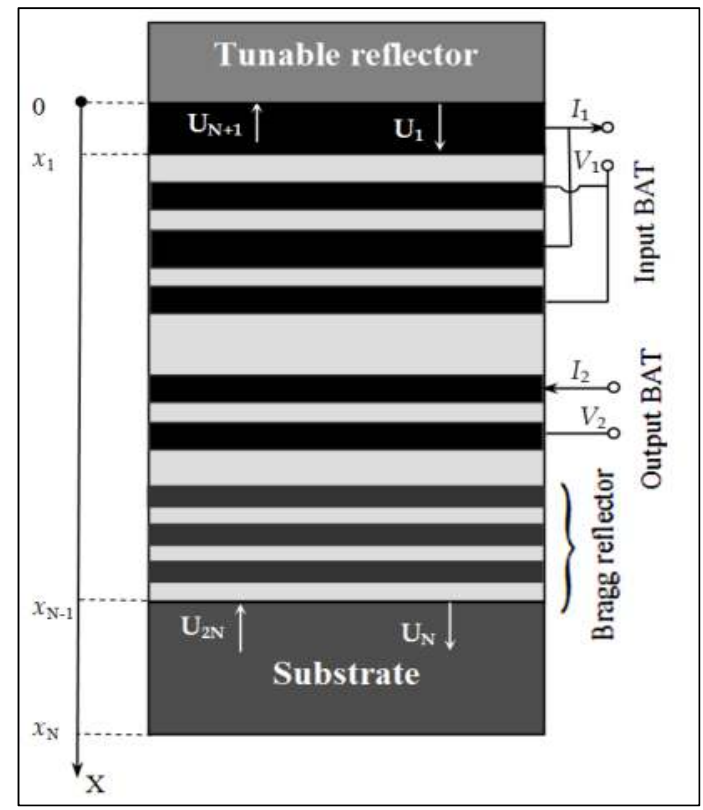

Figure 13. Schematic image of two-port solidly mounted BAW device.

The wave amplitudes $\mathrm{U} 1_{1 \ldots 2 \mathrm{~N}} \& \mathrm{U} 22_{1 \ldots 2 \mathrm{~N}}$ within domain $0 \leq x \leq x_{\mathrm{N}}$ on Fig.13 may be found in analogy with the previous analysis, brought in "Sect. 3.3", when voltage is applied either to input or output transducers $\left(V_{1}=1 \& V_{2}=0 \Rightarrow \mathrm{U}_{\mathrm{m}}=\mathrm{U} 1 \mathrm{~m} ; V_{1}=0 \& V_{2}=1 \Rightarrow \mathrm{U}_{\mathrm{m}}=\mathrm{U} 2 \mathrm{~m}\right)$. 
A couple of changes should be, yet, involved into the modeling, when forming the general scattering matrix $\hat{M}$ of SMR in whole (besides, surely, the novel spatial distribution of materials and thickness of layers there). First, $\tilde{P}_{22}$-parameter of the "top side" tunable reflector, found under condition $B_{2}=0$, must be used as the $1^{\text {st }}$ term in the sub-matrix $\hat{M} 12$ :

$$
\hat{\mathrm{M}} 12_{11}=\tilde{P}_{22}\left(Y_{0}\right) \cdot B C_{1}
$$

Secondly, $\hat{\mathrm{M}} 21_{\mathrm{NN}}=B C_{2} \cdot e x_{N}^{-4}$. Here $B C_{1,2}$ are the components of the boundary condition vector, relating to the domain $x \in\left[0, x_{\mathrm{N}}\right]$ on Fig.13 ( $B C_{2}=-1$ means, e.g., the rigidly clumped bottom side of a substrate).

Then, using (57), one has to determine afresh the corresponding distribution of electrical displacements within the transducers for the voltages applied either to input or output MBTs, finding all the needed $Y$ - parameters of arbitrary four-terminal network:

$$
I_{1,2}=Y_{11,22} \cdot V_{1,2}+Y_{12,21} \cdot V_{2,1}
$$

Accordingly to the energy balance condition at every frequency point of analysis and for arbitrary architecture of a device the normalized acoustic power hypothetically going outside a system (when $\left.B C_{1} \cdot B C_{2}=0\right)$ under external voltage, applied either to input $\left(P_{\mathrm{T}}=P \mathrm{a}_{1}\right)$ or output $\left(\mathrm{P}_{\mathrm{T}}=\mathrm{Pa}_{2}\right)$ ports, in the lossless case absolutely coincides with the input/output conductance values, and becomes smaller them in the presence of dissipation (a part of energy, supplied by source, is transformed to a heat):

$$
\begin{gathered}
\operatorname{Re}\left(Y_{11,22}(f)\right) \cdot\left|V_{1,2}\right|^{2} \geq P a_{1,2}(f) \text {, where } \\
\left\{\begin{array}{l}
P a_{1}=\omega^{2} \cdot S \cdot\left|V_{1}^{2}\right| \cdot\left\{\operatorname{Re}\left(Z_{N}\right) \cdot\left|U 1_{N}\right|^{2} \cdot\left(1-\left|B C_{2}\right|\right)+\operatorname{Re}\left(Z_{1}\right) \cdot\left|U 1_{N+1}\right|^{2} \cdot\left(1-\left|B C_{1}\right|\right)\right\}, \text { if } V_{2}=0 \\
P a_{2}=\omega^{2} \cdot S \cdot\left|V_{2}^{2}\right| \cdot\left\{\operatorname{Re}\left(Z_{N}\right) \cdot\left|U 2_{N}\right|^{2} \cdot\left(1-\left|B C_{2}\right|\right)+\operatorname{Re}\left(Z_{1}\right) \cdot\left|U 2_{N+1}\right|^{2} \cdot\left(1-\left|B C_{1}\right|\right)\right\}, \text { if } V_{1}=0
\end{array}\right.
\end{gathered}
$$

Note, as well, that one more checking rule must be applied here too. It is coupled with the reciprocity principle, being valid for any four-terminal network, based on acoustic waves:

$$
Y_{21}=Y_{12}
$$

The present model always passes through this test also - under the arbitrary combination of the topological and material parameters of a system.

Figures 14(a,b) illustrate how an input impedance of the one-port SMR $\left(Z_{e}=1 /\left|Y_{11}\right|\right)$ may be influenced by the variable reactance for some combinations of SMR's input data. Two variants (with more realistic thicknesses of electrodes) have been considered here, when the TRs contain a) one (with thickness $d p_{\mathrm{TR}} \approx 2.49 \mu \mathrm{m}$ ), and b) three (with thickness $d p_{\mathrm{TR}} \approx 2.57$ $\mu \mathrm{m})$ AlN layers. At this point BT contains in both cases a one piezoelectric layer with thickness $d p_{\mathrm{BT}}=\lambda_{\mathrm{AlN}} / 2 \approx 2.73 \mu \mathrm{m}$. Thickness of electrodes (with area $S=1 \mathrm{~mm}^{2}$ ) assumed to 
be different within TR $($ te $\mathrm{TR} \approx 0.16 \mu \mathrm{m})$ and $\mathrm{BT}($ te $\mathrm{BT} \approx 1.58 \mu \mathrm{m})$. The Bragg reflector, containing $\mathrm{Nr}=10$ "quarter-wavelength" layers, made from $\mathrm{SiO}_{2} \&$ Mo films, separates a transducer from the bottom substrate (Si) which has a thickness about $1 \mathrm{~mm}$.

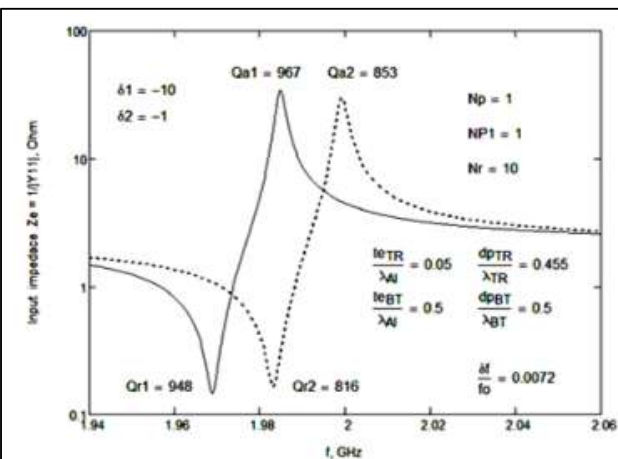

(a)

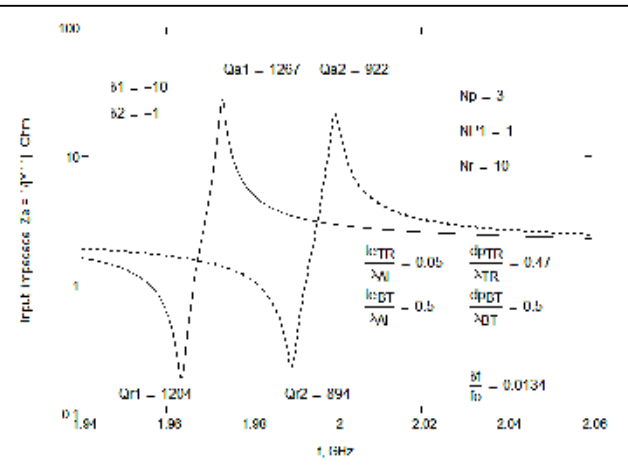

(b)

Figure 14. Input impedance of SMR with the rigidly clamped bottom surface of a substrate (Si). Tunable reflector is loaded only by variable capacitor, allowing changes of its capacitance From $\widetilde{C}=9 \cdot C_{0}$ (solid lines) to $\widetilde{C}=0$ (dotted lines), where $C_{0}$ is a static capacitance of TR. Calculations are made in two cases, when TR contains one (a) and three (b) AlN layers.

As one can see, the SMR's $Q$-factors at both resonant $\left(Q_{r}\right)$ and anti-resonant $\left(Q_{a}\right)$ frequencies increase when utilizing a multi-layer tunable reflector. Even these (not optimized yet) variant shows that under $N p=3$ a fractional interval of the frequency tuning reaches a rather large quantity $\left(\delta f / f_{0} \approx 1.34 \%\right.$ ), to be almost twice better than for SMR with a "one layer" TR. At this time only a capacitive reactance (varicap with Q-factor equaled to 100) is assumed to be used as a load, in order to prevent increasing of Ohm losses, which rise usually when using an inductor in the external electrical circuitry.

\section{Conclusion}

A highly efficient self-consistent analytical model, allowing us to describe an arbitrary BAW device, has been developed. Comprehensive solution of several typical tasks is given with the clear physical argumentation. Flexible one-dimensional modelling is based on a direct solution of the motion and constitutive acoustic equations, taking into account the relevant boundary conditions. Any kind of dissipation may be involved into consideration at the phenomenological level.

Using the proposed approach one may analyze and synthesize, while remaining within the frame of the same investigation manner, a structure with an arbitrary number and sequence of dielectric and metal layers. Multiple electrodes may compose the multilayer transducers forming those based one- and two-port networks. 
A method to control over the resonant frequency of solidly mounted BAW resonators, based on the usage of multi-layered tunable reflectors, is investigated in detail. It improves substantially the SMR tunability and may be applied either with the aim to correct for frequency errors, caused by technological thickness variations of layers, or in order to compensate the temperature drifts of the device characteristics using variable electrical load.

An original and very useful integral method is proposed to verify the validity of the simulation, when basing on three fundamental principles, namely: energy balance, the second law of thermodynamics, and reciprocity. The presented checking algorithm, on the one hand, gives us assurance in the modeling correctness. On the other hand, it simplifies considerably the search for mistakes during preparation of the corresponding software tools needed to optimize the device parameters in the shortest time.

\section{Author details}

Boris Sveshnikov

Lebedev Research Center in Physics, Moscow, Russia

Sergey Nikitov

Institute of Radio-engineering and Electronics of RAS, Moscow, Russia

Sergey Suchkov

State University, Saratov, Russia

\section{Acknowledgements}

This work was supported by the Russian Government Grant \# 11.G34.31.0030.

The authors thank Ms. Tatyana Sveshnikova for helping with grammar editing of the text.

\section{References}

Giraud, S.; Bila, S.; Aubourg, M. \& Cros, D. (2007). Bulk acoustic wave resonators 3D simulation, 2007 Joint with the 21st European Frequency and Time Forum, pp. 1147-1151, IEEE International Digital Object Identifier: 10.1109/FREQ.2007.4319258

Bradley, P.; Ruby, R.; Barfknecht, A.; Geefay, F.; Han, C.; Gan, G.; Oshmyansky, Y. \& Larson, J. (2002). A $5 \mathrm{~mm} \times 5 \mathrm{~mm} \times 1.37 \mathrm{~mm}$ Hermetic FBAR Du-plexer for PCS Handsets with Wafer-Scale Packaging, IEEE Ultrasonics Symposium Proceedings, 2002, pp. 931-934, ISSN : 1051-0117

Ruby R., Review and Comparison of Bulk Acoustic Wave FBAR, SMR Technology, IEEE Ultrasonics Symposium Proceedings, 2007, pp. 1029-1040

Fattinger, G. (2008), BAW Resonator Design Considerations - An Overview, IEEE Ultrasonics Symposium Proceedings, 2008, pp. 762-767

Hashimoto, K. (Ed.). (2009). RF Bulk Acoustic Wave Filters for Communications, ARTECH HOUSE, ISBN-13: 978-1-59693-321-7, Norwood, MA 02062 
Ballato, A.; Bertoni, H. \& Tamir, T. (1974). Systematic design of stacked-crystal filters by microwave network methods, IEEE Trans. Microwave Theory Tech., MTT-22, pp. 14-25, 1974

Novotny, H. \& Benes, E. (1991). Layered piezoelectric resonators with an arbitrary number of electrodes (general one-dimensional treatment), Journ. Acoust. Soc. Am., Vol. 90, Sept. 1991, pp. 1238-1245

Auld, B. (1973). Acoustic Fields and Waves in Solids, Vol. 1., New York: John Wiley \& Sons, Inc., 1973

Kino, G. (1987). Acoustic waves: devices, imaging, and analog signal processing, New Jersey: Prentice-Hall, 1987

Sveshnikov, B. (2009). Universal Modeling of the Bulk Acoustic Wave Devices, Proceedings of the EFFT-IFCS, 2009, pp. 466-469

Gulyaev Yu. \& Mansfeld G. (2004). Resonators and filters for microwave bulk acoustic wave devices - current status and trends, Uspekhi sovremennoi radioelectroniki, Moscow, 2004, \# 5-6, pp. 13-28 (in Russian).

Vorobiev, A . \& Gevorgian, S. (2010). Tunable thin film bulk acoustic wave resonators with improved Q factor, Appl. Phys. Lett., Vol. 96, no. 21, art. no. 212904, 2010.

Gristchenko, E. (1975). Acoustic analog of the electro-optical gate, Akust. Zh., Vol.21, no. 5, pp. 827-828 (in Russian)

Smith, W.; Gerard, R.; Collins, J.; Reeder, T. \& Shaw, H. (1969). Analysis of inter- digital surface wave transducers by use of an equivalent circuit model, IEEE Trans. On MTT, Vol. MTT-17, pp. 856-864, 1969

Sandler, M. \& Sveshnikov, B. (1981). An investigation of interdigital transducers of surface acoustic waves, taking into account the finite mass of the electrodes, Radio Engng. and Electron. Phys., vol. 26, No. 9, pp. 9-17, 1981.

Paskhin, V.; Sandler, M. \& Sveshnikov, B. (1981). A method to suppress the triple-transit signals in SAW filters, Zk Tekh. Fiz., Vol. 51, no. 12, pp. 2595-2597, (in Russian)

Sveshnikov, B. \& Filinov, V. (1988). Tunable SAW phase-shifting reflector using unidirectional IDT, Sov. Tech. Phys. Lett., v. 14, no. 8, pp. 658-660, 1988

Sveshnikov, B. (1995). Tunable Phase-Shifting Reflectors of Surface and Bulk Acoustic Waves, Ultrasonics World Congress Proceedings, Berlin, 1995, pp. 387-390.

Kucheryavaya, E.; Mansfeld, G.; Sveshnikov, B. \& Freik, A. (1995). Frequency control of composite volume acoustic-wave resonators, Acoustical physics, 41(2), 1995, pp. 302-304

Defaÿ, E.; Hassine, N.; Emery, P.; Parat, G.; Abergel, J. \& and Devos, A. (2011). Tunability of aluminum nitride acoustic resonators: A phenomenological approach, IEEE Transactions on UFFC, Vol. 58, no. 12, December 2011, pp. 2516-2520 